\title{
Contribution of Sensory Encoding to Measured Bias
}

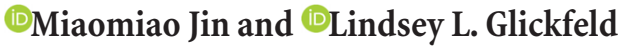 \\ Department of Neurobiology, Duke University Medical Center, Durham, North Carolina 27710
}

Signal detection theory (SDT) is a widely used theoretical framework that describes how variable sensory signals are integrated with a decision criterion to support perceptual decision-making. SDT provides two key measurements: sensitivity $\left(d^{\prime}\right)$ and bias $(c)$, which reflect the separability of decision variable distributions (signal and noise) and the position of the decision criterion relative to optimal, respectively. Although changes in the subject's decision criterion can be reflected in changes in bias, decision criterion placement is not the sole contributor to measured bias. Indeed, neuronal representations of bias have been observed in sensory areas, suggesting that some changes in bias are because of effects on sensory encoding. To directly test whether the sensory encoding process can influence bias, we optogenetically manipulated neuronal excitability in primary visual cortex (V1) in mice of both sexes during either an orientation discrimination or a contrast detection task. Increasing excitability in V1 significantly decreased behavioral bias, whereas decreasing excitability had the opposite effect. To determine whether this change in bias is consistent with effects on sensory encoding, we made extracellular recordings from V1 neurons in passively viewing mice. Indeed, we found that optogenetic manipulation of excitability shifted the neuronal bias in the same direction as the behavioral bias. Moreover, manipulating the quality of V1 encoding by changing stimulus contrast or interstimulus interval also resulted in consistent changes in both behavioral and neuronal bias. Thus, changes in sensory encoding are sufficient to drive changes in bias measured using SDT.

Key words: contrast; mouse visual cortex; optogenetics; orientation; psychophysics; signal detection theory

\section{Significance Statement}

Perceptual decision-making involves sensory integration followed by application of a cognitive criterion. Using signal detection theory, one can extract features of the underlying decision variables and rule: sensitivity $\left(d^{\prime}\right)$ and bias $(c)$. Because bias is measured as the difference between the optimal and actual criterion, it is sensitive to both the sensory encoding processes and the placement of the decision criterion. Here, we use behavioral and electrophysiological approaches to demonstrate that measures of bias depend on sensory processes. Optogenetic manipulations of V1 in mice bidirectionally affect both behavioral and neuronal measures of bias with little effect on sensitivity. Thus, changes in sensory encoding influence bias, and the absence of changes in sensitivity do not preclude changes in sensory encoding.

\section{Introduction}

Perceptual decision-making is a multistep process through which sensory information about the external world is first transformed into a neuronal code and then used to make a behavioral choice. In this process, both sensory encoding (i.e., the process by which a decision variable is generated) and the cognitive aspects of the

\footnotetext{
Received Jan. 10, 2019; revised April 17, 2019; accepted April 18, 2019.

Author contributions: M.J. and L.L.G. designed research; M.J. performed research; M.J. analyzed data; M.J. wrote the first draft of the paper; M.J. and L.L.G. edited the paper.

This work was supported by a NIH Director's New Innovator Award (DP2-EY025439), the Pew Biomedical Trusts, and the Alfred P. Sloan Foundation (L.L.G.). We thank B. Gincley and J. Sims for assistance with behavioral training; K. Leonard, M. Fowler, J. Isaac, and K. Murgas for surgical assistance; Z. Xu for assistance with software development; S. Lisberger, G. Field, G. Horwitz, C. Hull, and members of the Hull and Glickfeld laboratories for helpful discussions and comments on this paper.

The authors declare no competing financial interests.

Correspondence should be addressed to Lindsey L. Glickfeld at glickfeld@neuro.duke.edu.

https://doi.org/10.1523/JNEUROSCI.0076-19.2019

Copyright $\odot 2019$ the authors
}

decision-making process (i.e., the selection and application of a decision rule) are critical factors that determine the final choice (Gold and Shadlen, 2007; Carandini and Churchland, 2013; Romo and de Lafuente, 2013; Hanks and Summerfield, 2017).

Efforts to dissect the relative contribution of sensory and cognitive processes to decision-making often take advantage of signal detection theory (SDT), a classical and widely used framework that allows inference of the underlying decision variable distributions (e.g., signal and noise) and decision rule from behavioral measures (Green and Swets, 1966). In particular, SDT allows the use of hit and false alarm (FA) rates to extract two aspects of the perceptual decision: sensitivity $\left(d^{\prime}\right)$ and bias $(c)$. Measures of sensitivity allow inference of the separability of the underlying decision variable distributions of signal and noise (or target and distractor). Thus, this measure is thought to reflect the quality of encoding and integration in sensory circuits that provide input to the decision-making circuits (Bashinski and Bacharach, 1980; Bennett et al., 2013; Pinto et al., 2013; Luo and 
A

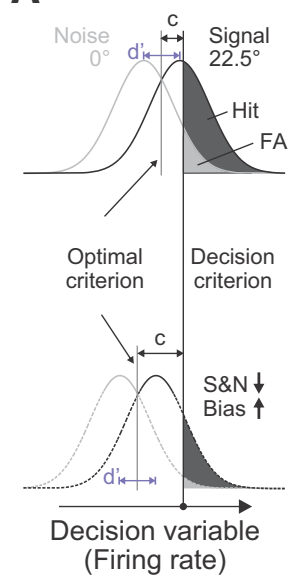

B

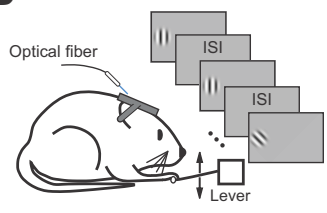

D

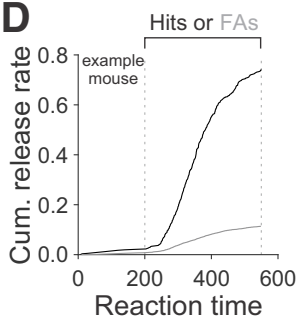

from target or distractor (ms)

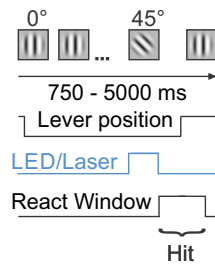

C

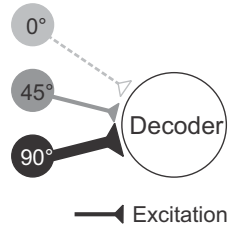

E
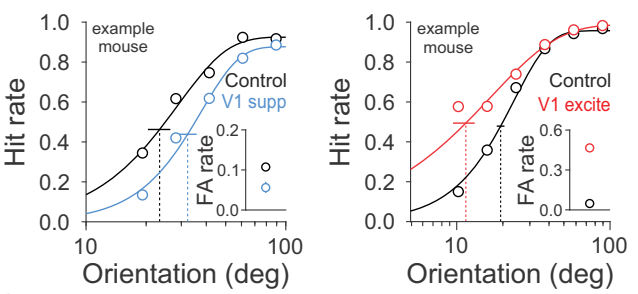

Figure 1. Optogenetically suppressing or exciting V1 decreases or increases hit and FA rates in an orientation discrimination task. $A$, Schematic of effect of shifting signal and noise distributions on bias measured using signal detection theory. Top, Distributions of target $\left(22.5^{\circ} ;\right.$ solid black, signal) and distractor $\left(0^{\circ} ;\right.$ solid gray, noise) responses. Note that bias (c) is measured as the distance between the actual (black vertical line) and optimal ( $c=0$; gray vertical line) criterion. Bottom, Manipulations that decrease both the target and distractor distributions shift the optimal criterion to the left, and therefore result in an increase in bias. Purple lines indicate the measured sensitivity $\left(d^{\prime}\right)$ that is the distance between the mean of target and distractor distributions. $B$, Schematic of behavior setup and trial progression. Blue light is turned on for a single target or distractor presentation on each trial. V1 suppression (blue) and excitation (red) is achieved via optogenetically driving PV + or VGAT + neurons and Emx1 + neurons respectively. C, Schematic of perceptual choice circuit for the orientation discrimination task. Orientation tuned excitatory neurons (shades of gray) converge onto the decoder with weights biased toward target-preferring neurons (modified from Jin et al., 2019). D. Cumulative release rate [as fraction of all targets (black) or distractor (gray) presentations] as a function of reaction times relative to the onset of target or distractor stimulus for an example mouse in the control condition. Vertical lines represent the react window used to calculate both hit and FA rate. E, Hit rate and FA rate (inset) for control (black) and V1 suppression (blue; left) or excitation (red; right) for one example mouse each. Note that the example mouse for V1 suppression is the same as in $\boldsymbol{D}$. Hit rates are fit with a Weibull function; vertical dotted lines are threshold, error is $95 \%$ confidence interval.

Maunsell, 2015; Jurjut et al., 2017; Ni et al., 2018). On the other hand, bias measures the overall tendency to classify the stimulus as signal or noise. Thus, it can reflect the subject's decision criterion. In fact, $c$ is often used synonymously with "criterion" and is therefore commonly thought to reveal cognitive contributions to the decision-making process and involve areas downstream of sensory cortex (McDonald et al., 2000; Grove et al., 2012; Jones et al., 2015; de Gee et al., 2017; Crapse et al., 2018; Luo and Maunsell, 2018; van Vugt et al., 2018).

Although changes in bias can result from a change in the subject's decision strategy, bias is the difference between the optimal and actual decision criterion, and thus changes in sensory encoding that shift the optimal criterion will result in a change in bias even when there is no change in decision criterion (Fig. 1A). This could happen when changes in the signal and noise distributions are not opposite and proportional (Witt et al., 2015). Indeed, neuronal correlates of bias have been identified in sensory cortical areas. Human neuroimaging experiments have found a strong correlation between the strength of representation of prior information (such as expected stimulus features or locations) in sensory areas and the strength of behavioral bias (White et al., 2012; Kok et al., 2013; Vintch and Gardner, 2014). Similarly, spontaneous fluctuations in the excitability of sensory cortical areas correlate with spontaneous fluctuations in behavioral bias (Iemi et al., 2017). Although these data suggest that activity in sensory areas can influence behavioral bias, these correlational studies cannot rule out the possibility that these effects are due to feedback from decision-making areas.

Although cognitive factors are clearly an important determinant of bias, we propose that at least some changes in bias are due to effects on sensory encoding. To directly test this hypothesis, we generated a behavioral paradigm in which we could address the causal relationship between activity in sensory areas and measured bias: a go/no-go orientation discrimination task in which mice need to discriminate target from distractor orientations (Fig. 1B). This task allows measures of hit and FA rates, and therefore bias and sensitivity. Importantly, in previous work we have identified the computation, and therefore the decision variable, that the mice use to solve this task: instead of optimally estimating the stimulus orientation from the activity of a tuned population, the decision-making circuit linearly sums the activity in visual cortical circuits, with higher weight given to neurons that prefer targets while ignoring those that prefer the distractor (Fig. 1C; Jin et al., 2019). Therefore, the decision variable and decision criterion can be approximated in units of firing rate. Thus, manipulations that coincidently alter firing rates in response to targets and distractors will shift the signal and noise distributions in the same direction, change the optimal criterion, and induce a change in measured bias (Fig. 1A).

Indeed, we found that altering neuronal responses to targets and distractors through either direct optogenetic manipulation of neurons in primary visual cortex (V1) or manipulation of visual stimulus properties resulted in a reliable change in behavioral bias with relatively little impact on sensitivity. Further, electrophysiological recordings from neurons in V1 during each of these manipulations also revealed a strong effect on bias in the same direction as during behavior. Thus, changes in bias can be driven by changes in either cognitive factors or sensory encoding, and the lack of a change in sensitivity does not preclude a change in sensory encoding.

\section{Materials and Methods}

Animals. All animal procedures conformed to standards set forth by the $\mathrm{NIH}$, and were approved by the IACUC at Duke University. Twentyseven mice [both sexes; 3-24 months old; singly and group housed (1-4 in a cage) under a regular $12 \mathrm{~h}$ light/dark cycle; C57/B6J (Jackson Laboratories, 000664) was the primary background with up to $50 \% \mathrm{CBA} / \mathrm{CaJ}$ (Jackson Laboratories, 000654)] were used in this study. Pvalb-cre 
(tm1 (cre)Arbr; Jackson Laboratories, 008069; $n=17$; PV::Cre), VGATChR2-EYFP (Slc32a1-COP4 ${ }^{*}$ H134R/EYFP; Jackson Laboratories, 014548; $n=$ 4) and Emx1-IRES-Cre (tm1 (cre)Krj; Jackson Laboratories, 005628; $n=$ 6; EMX1::Cre) were crossed to C57/B6J mice for in vivo extracellular electrophysiology $(n=11)$ and behavior $(n=18)$ experiments. Note that two of the mice (one PV::Cre and one Emx1::Cre) were used in both behavior and recording.

Cranial window implant. Dexamethasone $(3.2 \mathrm{mg} / \mathrm{kg}$, s.c.) and Meloxicam $(2.5 \mathrm{mg} / \mathrm{kg}$, s.c. $)$ were administered at least $2 \mathrm{~h}$ before surgery. Animals were anesthetized with ketamine ( $200 \mathrm{mg} / \mathrm{kg}$, i.p.), xylazine (30 $\mathrm{mg} / \mathrm{kg}$, i.p.), and isoflurane $\left(1.2-2 \%\right.$ in $\left.100 \% \mathrm{O}_{2}\right)$. Using aseptic technique, a headpost was secured using cyanoacrylate glue and C\&B Metabond (Parkell), and a $5 \mathrm{~mm}$ craniotomy was made over the left hemisphere (center: $2.8 \mathrm{~mm}$ lateral, $0.5 \mathrm{~mm}$ anterior to lambda) allowing implantation of a glass window (an $8 \mathrm{~mm}$ coverslip bonded to two $5 \mathrm{~mm}$ coverslips (Warner, no. 1) with refractive index-matched adhesive (Norland, no. 71) using Metabond.

The mice were allowed to recover for 1 week before habituation to head restraint. Habituation to head restraint increased in duration from 15 min to $>2$ h over 1-2 weeks. During habituation and electrophysiology sessions, mice were head restrained while allowed either to freely run on a circular disc (InnoWheel, VWR) or to rest in a plastic tube.

Retinotopic mapping. Retinotopic maps generated from intrinsic autofluorescence or cortical reflectance (for VGAT-ChR2-EYFP mice). For intrinsic autofluorescence, the brain was illuminated with blue light (473 $\mathrm{nm}$ LED, Thorlabs) or a white light source (EXFO) with a $462 \pm 15 \mathrm{~nm}$ bandpass filter (Edmund Optics), and emitted light was measured through a green and red filter (500 nm long-pass); for cortical reflectance, the brain was illuminated with orange light (530 $\mathrm{nm}$ LED, Thorlabs), and all of the reflected light was collected. Images were collected using a CCD camera (Rolera EMC-2, Qimaging) at $2 \mathrm{~Hz}$ through a $5 \times$ air-immersion objective ( 0.14 numerical aperture, Mitutoyo), using Micromanager acquisition software (NIH). Stimuli were presented at $4-6$ positions (drifting, sinusoidal gratings at $2 \mathrm{~Hz}$ ) for $10 \mathrm{~s}$, with $10 \mathrm{~s}$ of mean luminance preceding each trial. Images were analyzed in ImageJ (NIH) to measure changes in fluorescence $(\mathrm{dF} / \mathrm{F}$; with $F$ being the average of all frames) to identify V1 and the higher visual areas. Vascular landmarks were used to identify targeted sites (V1) for electrophysiology and optogenetics experiments.

Viral injection. We targeted V1 in PV::Cre mice $(n=6)$ for expression of Channelrhodopsin2 (ChR2) and in Emx1::Cre mice $(n=6)$ for expression of Chronos. Dexamethasone $(3.2 \mathrm{mg} / \mathrm{kg}$, s.c.) was administered at least $2 \mathrm{~h}$ before surgery and animals were anesthetized with isoflurane $\left(1.2-2 \%\right.$ in $\left.100 \% \mathrm{O}_{2}\right)$. The coverslip was sterilized with $70 \%$ ethanol and the cranial window removed. A glass micropipette was filled with virus [AAV5.EF1.dFloxed.hChR2.YFP (titer: $3.74 \mathrm{e} 12 \mathrm{GC} / \mathrm{ml}$; UPenn, CS0384), AAV9.hSyn.FLEX.rc.Chronos.GFP (titer: 1.25e12 GC/ml; Addgene, 59056), or AAV2/9.CAGGS.FLEX.ChR2.tdTomato (titer: $2.44 \mathrm{e} 12$ GC/ml; Addgene, 18917)], mounted on a Hamilton syringe, and lowered into the brain. Fifty nanoliters of virus were injected at 250 and $500 \mu \mathrm{m}$ below the pia $(30 \mathrm{nl} / \mathrm{min})$; the pipette was left in the brain for an additional $10 \mathrm{~min}$ to allow the virus to infuse into the tissue. Following injection, a new coverslip was sealed in place, and for behavioral experiments, an optical fiber (400 $\mu \mathrm{m}$ diameter; Doric Lenses) was attached to the cranial window above the injection site. Optogenetic behavioral experiments and electrophysiology experiments were conducted at least 2 weeks following injection to allow for sufficient expression.

Behavioral task. Animals were water scheduled and trained to discriminate the orientation of visual stimuli or detect the appearance of a visual stimulus by manipulating a lever. The behavior training and testing occurred during the light cycle.

For the orientation discrimination task, we first trained mice to detect a full-field, $90^{\circ}$ orientation difference (target) from a static grating (distractor). Most mice $(n=12)$ were trained with a $0^{\circ}$ (vertical) distractor; however, two mice were trained with a $45^{\circ}$ (counterclockwise from vertical) distractor. On the initial days of training, mice were rewarded for holding the lever for at least $400 \mathrm{~ms}$ (required hold time) but not $>20 \mathrm{~s}$ (maximum hold time). At the end of the required hold time, the grating changed orientation $\left(90^{\circ}\right.$ counterclockwise difference from the distrac- tor) until the mouse released the lever or the maximum hold time expired. Typically, within 2 weeks of training, the mice began releasing the lever as soon as the target orientation appeared. Once the animals began reliably responding to the target orientation, we added a random delay between lever press and target stimulus to discourage adoption of a timing strategy. Over the course of the next few weeks, the task was made harder by (in roughly chronological order): (1) increasing the random delay, (2) decreasing the target stimulus duration and reaction time window, (3) removing the stimulus during the intertrial interval (ITI), (4) shrinking and moving the stimuli to more eccentric positions, (5) adding a mean-luminance interstimulus interval (ISI) to mask the motion signal in the orientation change, and finally (6) introducing hard targets (range: $\left.9-90^{\circ}\right)$ to probe discrimination threshold. Delays after errors were also added to discourage lapses (missing the easiest target; i.e., $90^{\circ}$ ) and early releases (releases before the reaction time window).

In the final form of the task, each trial was initiated when the ITI $(3 \mathrm{~s})$ had elapsed and the mouse had pressed the lever. Trial start triggered the presentation of a series of $100 \mathrm{~ms}$ static sinusoidal, Gabor patches [diameter: $30^{\circ}$, spatial frequency (SF): $0.1 \mathrm{cycle} /{ }^{\circ}$, contrast: $100 \%$, positioned at an eccentricity of $30-40^{\circ}$ in azimuth and $0-10^{\circ}$ in elevation] followed by a target orientation of the same parameters but of a different orientation. The target orientation occurred with a variable delay (flat distribution) after at least two distractor presentations (up to 9 distractors). Following each target, additional distractors were presented until either the mouse released the lever or the reaction time expired. Within each trial, each stimulus presentation (distractors and target) was separated by a meanluminance ISI $(250,500$, or $750 \mathrm{~ms})$. Some variables were randomized on a trial-by-trial basis (e.g., number of distractor presentations and target orientation), whereas other variables were randomized within a trial on a presentation-by-presentation basis (e.g., ISI). Each trial had the possibility of having a target presentation, if the mouse held the lever through the all of the preceding distractor presentations. Mice received water reward only if they released the lever within 100-650 ms (sometimes extended to $1000 \mathrm{~ms}$ ) after a target occurred. If mice released the lever before reaction time began (early release) or failed to release the lever by the time the reaction time expired (miss), the trial would be aborted and additional time (2-4 s) would be added to the ITI.

For a subset of mice ( $n=5$; see Fig. 6$)$, the contrast of each presentation was also randomized (Michelson contrast: 30,50 , and $70 \%$ ) on a presentation-by-presentation basis; in these experiments the stimulus size was reduced to $20^{\circ}$ and the $\mathrm{SF}$ increased to 0.16 cycle $/{ }^{\circ}$ to reduce the contribution of surround suppression and therefore linearize the contrast-response functions (Sengpiel et al., 1997; Nienborg et al., 2013). To compensate for the difficulty induced by low contrast and small size of the stimuli, we presented the task stimuli at a more central location $\left(5-15^{\circ}\right.$ in azimuth and $10^{\circ}$ in elevation).

For the contrast detection task (see Fig. 5), we first trained mice to detect a full-field, $100 \%$ contrast, $0^{\circ}$ static target grating from mean luminance (gray) screen that appeared at the end of the required hold time (400 ms). Once the animals started to respond to the appearance of the target grating stimulus by releasing the lever, we gradually made the task harder by: (1) increasing the random delay (final max value: $3.5 \mathrm{~s}$ ) between the lever press and a target grating appearance; (2) shrinking and moving the stimuli to more eccentric positions $\left(30^{\circ}\right.$ in diameter, $\mathrm{SF}$ of 0.1 cycle $/^{\circ}$, positioned at an eccentricity of $30-40^{\circ}$ in azimuth and $0-10^{\circ}$ in elevation); and (3) introducing hard targets (range: $4-100 \%$ contrast) to probe contrast detection threshold. Delays after errors were also added to discourage lapses and early releases.

For optogenetic stimulation (Figs. 1, 3, and 5), we delivered blue light to the brain though a 400 micrometer optic fiber from a $473 \mathrm{~nm}$ LED (Thorlabs) or a $450 \mathrm{~nm}$ laser (Optoengine) and calibrated the total light intensity out of the fiber. For the orientation discrimination task, the light power was titrated so that it did not induce significant changes in the lapse rate. For V1 suppression, the mean light power was $0.27 \pm 0.07 \mathrm{~mW}$ (range: $0.07-0.4 \mathrm{~mW}$ ); and for $\mathrm{V} 1$ excitation, the mean light power was $0.06 \pm 0.02 \mathrm{~mW}$ (range: $0.02-0.1 \mathrm{~mW}$; Figs. 1,3 ). On each trial, a single stimulus (either the distractor two stimuli before the target, the distractor before the target, the target, or the distractor after the target) was targeted with equal probability. The light was turned on $\sim 30 \mathrm{~ms}$ before the time of 
Table 1. Mice overlap and timeline among three datasets

\begin{tabular}{lllll}
\hline Mouse ID & Genotype & Optogenetics & Contrast & $|S|$ \\
\hline a & EMX1::Cre & 1 & 0 & 0 \\
b & EMX1::Cre & 1 & 2 & 0 \\
c & VGAT-ChR2 & 1 & 2 & 0 \\
d & EMX1::Cre & 2 & 0 & 1 \\
e & EMX1::Cre & 2 & 0 & 1 \\
$\mathrm{f}$ & PV::Cre & 2 & 0 & 1 \\
g & VGAT-ChR2 & 2 & 0 & 1 \\
h & PV::Cre & 3 & 2 & 1 \\
i & PV::Cre & 0 & 2 & 1 \\
j & PV::Cre & 0 & 2 & 1 \\
k & PV::Cre & 0 & 2 & 1 \\
I & PV::Cre & 0 & 0 & 1 \\
$\mathrm{~m}$ & PV::Cre & 0 & 0 & 1 \\
$\mathrm{n}$ & PV::Cre & 0 & 0 & 1 \\
\hline
\end{tabular}

visual presentation onset for the duration of the stimulus (100 ms). For the contrast detection task, the light stimulation was on for the entire duration of a trial on $50 \%$ of trials with the mean power $0.36 \pm 0.04 \mathrm{~mW}$ (range: $0.25-0.4 \mathrm{~mW}$; Fig. 5). Behavioral control and stimulus presentation used MWorks (http://mworks-project.org), and custom software in MATLAB (MathWorks).

Notably, there are overlapping animals in the datasets for the optogenetic (Figs. 1, 3), contrast (see Fig. 6), and ISI manipulations (see Fig. 7) for the orientation discrimination task. Table 1 describes the overlap and difference in time in collecting these datasets. Numbers (1-3) indicate the time sequence in which the data were collected for each mouse, whereas 0 reflects no training on that task. Four mice were trained in a single task, nine mice were trained on two tasks (thus belonged to two datasets), and only one mouse was included in all datasets. Notably, all four mice in the contrast detection task (see Fig. 5) are from a different cohort.

Visual stimulation. Visual stimuli were presented either on a $144 \mathrm{~Hz}$ (Asus) or $120 \mathrm{~Hz}$ (Samsung) LCD monitor, calibrated with an i1 Display Pro (X-rite), for electrophysiology and behavior experiments, respectively. The monitor was positioned $21 \mathrm{~cm}$ from the contralateral eye. Circular Gabor patches containing static sine-wave gratings alternated with periods of uniform mean luminance $\left(60 \mathrm{~cd} / \mathrm{m}^{2}\right)$.

Three visual stimulus protocols were used for electrophysiology experiments. Protocol 1 (Figs. 2, 4): Each trial began with six sequential static distractor presentations $\left(0^{\circ}\right.$; duration: $100 \mathrm{~ms}$; diameter: $30^{\circ}$; SF: 0.1 cycle $/^{\circ}$; contrast: $100 \%$; ISI: $250 \mathrm{~ms}$ ) followed by a target presentation of the same parameters but of different orientations $\left(22.5,45\right.$, or $\left.90^{\circ}\right)$. Optogenetic stimulation during Protocol 1 was matched to the behavioral conditions. Protocol 2 (see Fig. 6): similar to Protocol 1, except the contrast of each stimulus presentation was randomized from 30,50 , and $70 \%$. As in the behavior, the stimulus size was reduced to $20^{\circ}$ in diameter (SF: $0.16 \mathrm{cycle}^{\circ}{ }^{\circ}$ ) to limit the contribution of increasing surround suppression with increasing contrast. Protocol 3 (see Fig. 7): similar to Protocol 1, except: (1) the number of distractors was randomized from two to nine within each trial, and (2) the ISI between each presentation was randomized (250, 500, or $750 \mathrm{~ms})$. All protocols had an ITI of $4 \mathrm{~s}$.

Extracellular electrophysiology. Electrophysiological signals were acquired with a 32-site polytrode acute probe (either A4x8-5mm-100400-177-A32 (4 shanks, 8 site/shank at $100 \mu \mathrm{m}$ spacing) or A1x32-Poly25mm-50s-177-A32 (1 shank, 32 sites, $25 \mu \mathrm{m}$ spacing; NeuroNexus) through an A32-OM32 adaptor connected to a Cereplex digital headstage (Blackrock Microsystems). Unfiltered signals were digitized at 30 $\mathrm{kHz}$ at the headstage and recorded by a Cerebus multichannel data acquisition system (Blackrock Microsystems). Visual stimulation synchronization signals were also acquired through the same system via a photodiode directly monitoring LCD output.

On the day of recording, the cranial window (and the optic fiber, if it was already implanted from behavioral experiments) was removed, and a small durotomy performed to allow insertion of the electrode into V1. A ground wire was connected via a gold pin cemented in a burr hole in the anterior portion of the brain. The probe was slowly lowered into the brain (over the course of $15 \mathrm{~min}$ with travel length of $\sim 800 \mu \mathrm{m}$ ) until the most superficial recording site was in the brain and allowed to stabilize for 45-60 min before beginning recordings. For optogenetic stimulation in Protocol 1, the optic fiber was held in place via an articulated arm (Flexbar, SKU 14830) to allow light delivery (473 nm LED, Thorlabs) to the recording site. For V1 suppression, the mean light power was $0.28 \pm$ $0.02 \mathrm{~mW}$ (range: $0.1-0.4 \mathrm{~mW}$ ); and for V1 excitation, the mean light power was $0.05 \pm 0.003 \mathrm{~mW}$ (range: $0.03-0.06 \mathrm{~mW}$ ), matching the ranges that were used in the behavioral tests.

Of the 11 mice that were used for extracellular electrophysiology, 3 were previously trained in the orientation discrimination task, 3 were trained in a contrast discrimination task (not in this study), and 5 were naive. To test whether training history might influence our results, we compared bias measured from neuronal populations recorded in trained versus naive mice. The effects of optogenetic manipulation of bias was independent of whether the mice were previously trained [interaction between effects of $\mathrm{V} 1$ excitation and training history (naive vs trained) on bias: $p=0.51, \mathrm{DF}=1$; two-way ANOVA] or which task they were trained on [interaction between effects of V1 suppression and task (contrast vs orientation discrimination) for bias: $p=0.8, \mathrm{DF}=1$; two-way ANOVA].

\section{Data processing}

Behavior processing and analysis. All behavioral processing and analysis were performed in MATLAB. All trials were categorized as either an early release, hit, or miss based on the time of release relative to target onset: responses occurring earlier than $100 \mathrm{~ms}$ after the target stimulus were considered early releases; responses occurring between 200 and $550 \mathrm{~ms}$ after the target were considered hits; failures to respond before $550 \mathrm{~ms}$ after the target were considered misses. The same reaction window (200$550 \mathrm{~ms}$ ) was used following each distractor to calculate false alarm (FA) rate (thus FAs are a subset of early releases). This window as selected to ensure that the majority of the hits and FAs are because of stimulus driven responses to the immediately preceding stimulus (Fig. 1D) and there are independent reaction windows for adjacent stimuli with short ISIs (Jin et al., 2019). Thus, each distractor presentation was categorized as either a FA or correct reject (CR), and each target presentation was either a hit or a miss. Because there were no distractors presented in the contrast detection task (see Fig. 5), we calculated FA rate by simulating the timing of potential distractor presentations to match the potential timing of target presentations, and assessing the probability of the mouse releasing the lever during these windows.

Behavioral sessions were manually cropped to include only consecutive trials in each session with stable periods of performance. Sessions with optogenetic manipulations were selected based on the following criteria: (1) at least $40 \%$ of trials were hits, and $(2)<50 \%$ of trials were early releases. Sessions with stimulus manipulations were selected based on the following criteria: (1) at least $50 \%$ of trials were hits, and (2) $<35 \%$ of trials were early releases. Based on these criteria, the data in Figure 3, V1 suppression, included $16 \pm 3$ (range: $8-19$ ) sessions for each mouse with $4793 \pm 706$ trials (range: $3408-6695$ ); Figure 3, V1 excitation, included $29 \pm 13$ (range: $3-58$ ) sessions for each mouse with $8416 \pm$ 3981 trials (range: 1551-18,102); the data in Figure 5 included $36 \pm 14$ sessions (range: 5-60) for each mouse with an average of 13,275 \pm 5519 trials per mouse (range: $2084-25,302$ ); the data in Figure 6 included $34 \pm$ 13 (range: $11-75$ ) sessions for each mouse with $8975 \pm 3519$ trials (range: 1017-23,181); and the data in Figure 7 included $17 \pm 3$ sessions (range: 5-46) for each mouse with an average of $6348 \pm 815$ trials per mouse (range: 2593-11,857).

Hit rate was computed from the number of hits and misses for each stimulus type:

$$
\text { Hit rate }=\frac{\text { hit }}{\text { hit }+ \text { miss }} \text {. }
$$

FA rate was computed from the total number of FAs and CRs in the session:

$$
F A \text { rate }=\frac{F A}{F A+C R} .
$$


A
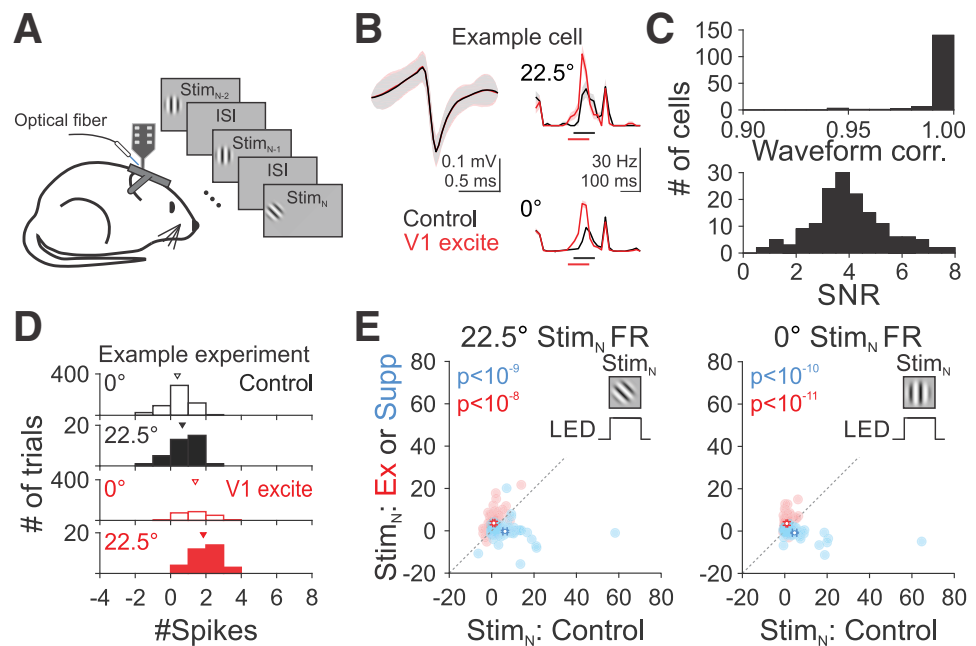

$E$
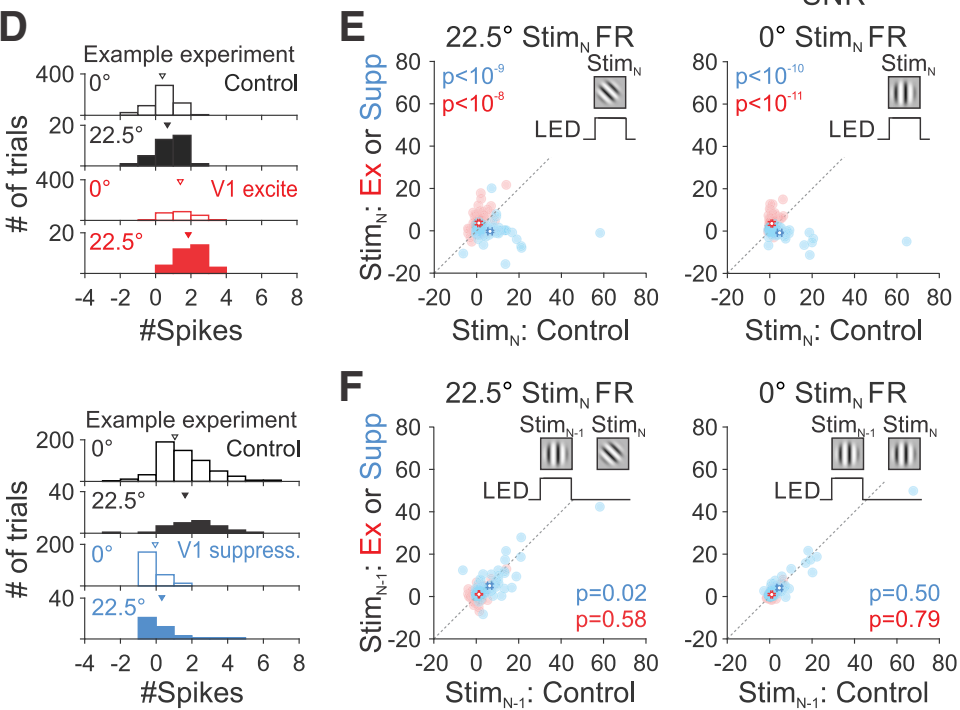

\section{$F$}

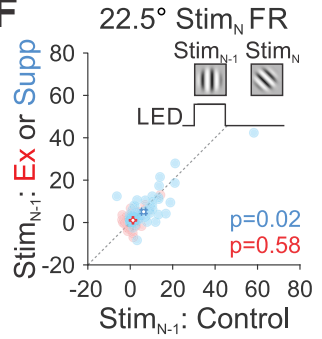

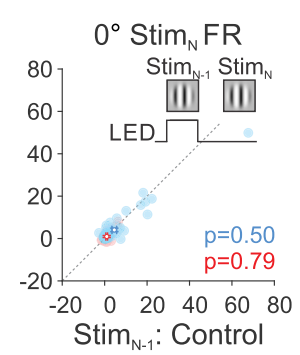

Figure 2. Optogenetically suppressing or exciting V1 decreases or increases neuronal responses to both targets and distractors. $\boldsymbol{A}$, Schematic of extracellular recording setup. Stimuli are presented as in Figure $1 \boldsymbol{B}$ to passively viewing mice. $\boldsymbol{B}$, Left, Mean waveform shapes for control and V1 excitation for and an example cell. Shaded area is SD across spikes. Right, Same cell's responses to $22.5^{\circ}$ target (top) and $0^{\circ}$ distractor (bottom) for control (black) and V1 excitation (red). Black horizontal bar is the duration of the visual presentation and red horizontal bar is the duration of light delivery for exciting V1. Shaded area is SEM across trials. C, Top, Histogram of the correlation coefficient of waveform shapes between control and V1 suppression/excitation. Bottom, Signal-tonoise ratio (SNR; mean/SD) of the trough value of the waveform across all the cells ( $n=153$ cells, including V1 suppression and excitation). $\boldsymbol{D}$, Distributions of spikes summed across a simultaneously recorded populations in response to distractor $\left(0^{\circ}\right.$; open bars) and target $\left(22.5^{\circ}\right.$; filled bars) stimuli on control trials (black) and during V1 excitation (red; $n=16$ cells; top) or suppression (blue; $n=17$ cells; bottom) for one example experiment each. Triangles show the mean of the distribution. $\boldsymbol{E}$, Comparison of neuronal responses (FR, in hertz) to the $22.5^{\circ}$ target (left) and $0^{\circ}$ distractor (right) between control and $\mathrm{V} 1$ excitation (red)/ suppression (blue) on the current stimulus $\left(S_{\mathrm{N}}\right.$ ). Filled circles are individual cells and error bars are $\mathrm{SEM}$ across cells with white dots in the center showing the mean of the population (excitation: $n=83$ cells, 3 mice; suppression: $n=70$ cells, 3 mice). $\boldsymbol{F}$, Comparison of neuronal responses (FR, in hertz) to the $22.5^{\circ}$ target (left) and $0^{\circ}$ distractor (right) on $\mathrm{Stim}_{\mathrm{N}}$ when the previous stimulus (Stim ${ }_{\mathrm{N}-1}$ ) was excited/suppressed versus control.

Signal detection theory (Green and Swets, 1966) was applied to measure behavioral sensitivity $\left(d^{\prime}\right)$ and bias $(c)$. Extreme values of hit and FA rate (i.e., 0 and 1$)$ were replaced with $0.5 / n$ and $(n-0.5) / n$, respectively, where $n$ is the number of target or distractor trials (Macmillan and Kaplan, 1985; Stanislaw and Todorov, 1999). $d^{\prime}$ and $c$ were then calculated as follows:

$$
\begin{gathered}
d^{\prime}=Z(H R)-Z(F A R), \\
c=-\frac{Z(H R)+Z(F A R)}{2},
\end{gathered}
$$

where $Z$ is the inverse of the cumulative distribution function of the normal Gaussian distribution; HR is hit rate, and FAR is FA rate.

Because the detection threshold varied across mice and not all the mice were tested at exactly the same orientations, the hit rate for $22.5^{\circ}$ is extrapolated based on a Weibull function fitted from the psychometric curve for each mouse.

Electrophysiology processing and analysis. Individual single units were isolated using the SpyKing CIRCUS package (http://spyking-circus. readthedocs.io/en/latest/). Raw data were first high-pass filtered $(>500 \mathrm{~Hz})$ and spikes were detected when a filtered voltage trace crossed threshold (9-13 median absolute deviations computed on each channel). A combination of density- based clustering and template matching algorithms was used to automatically cluster the spikes. The resulting clusters were then inspected and adjusted manually using a MATLAB GUI. Clusters with refractory period violations $(<2$ $\mathrm{ms},>1 \%$ violation) in the autocorrelogram and that were not stable across the whole recording session were discarded from the dataset. Clusters were combined if they met each of three criteria by inspection: (1) similar waveforms, (2) coordinated refractory periods in the cross-correlogram, and (3) similar ISI distribution shape. Unit position with respect to the recording sites was calculated as the average of all site positions weighted by the waveform amplitude of each site. For V1-suppression or -excitation experiments, we also quantified the similarity of the waveforms between control and optogenetic conditions using correlation coefficient $(r)$ values (Fig. 2C). Because for majority of the cells, V1 suppression strongly reduce firing rate rendering few or even no spikes for analyzing waveforms, we extended the window starting from $200 \mathrm{~ms}$ before visual onset, end with $250 \mathrm{~ms}$ after visual offset. Signal and noise ratio of the trough value of the waveform shape was calculated as mean divided by SD across spikes. All of the subsequent analysis was performed in MATLAB.

Visually-evoked responses of each unit in V1 were measured based on average peristimulus time histograms (PSTHs; bin size: $20 \mathrm{~ms}$; Fig. $2 B$ ) over repeated presentations ( $>25$ trials) of the same stimulus. Response amplitudes were measured on a trial-by-trial basis: by subtracting the firing rate at the time of the visual stimulus onset from the value at the peak of the average PSTH within a window of $0-100 \mathrm{~ms}$ after the visual onset. However, in the case of V1 excitation, responses were measured by subtracting the baseline firing rate (value at visual onset, bin $0 \mathrm{~ms}$, multiplied by 6 ) from the number of spikes during the visual presentation window $(0-100 \mathrm{~ms}, 6$ bins $)$. This is because the peak response latencies after V1 excitation were often shorter than the latencies of the visual responses in the control condition (Fig. 2B). "Responsive cells" were chosen as having statistically significant visually-evoked responses using a paired $t$ test to compare baseline responses (averaged over $0-100 \mathrm{~ms}$ before the visual onset) with visuallyevoked responses (averaged over $0-100 \mathrm{~ms}$ after the visual onset; this analysis window excluded off-responsive units from analysis). For V1suppression experiments, we excluded cells that were significantly driven by the light stimulation. For all protocols, we included cells that were significantly driven by either the first distractor stimulus or any of the target orientations. For Protocol 2, this test was only performed for the highest contrast stimuli. Thus, we included 70/110 cells for V1 suppression; and 83/109 cells for V1 excitation; for Protocol 2, we included 92/151 cells and for Protocol 3, 74/100 cells were included.

For calculation of predicted hit rate and FA rate: the distribution of single trial responses to the $22.5^{\circ}$ target was compared with the distribution of responses to the distractor $\left(0^{\circ}\right.$, third-sixth stimulus). For Protocol $1-\mathrm{V} 1$ suppression, the decision criterion for each cell was fixed as the mean of the responses to the suppressed distractor and the target in control trials (Fig. 4A). For Protocol 1-V1 excitation, the decision criterion for each cell was fixed as the mean of the responses to the distractor in control conditions and the excited target. We also chose alternative decision criterion values by using sliding criterion values ( 10 bins with equal distance between the maximum and minimal firing rates across 
conditions) to choose the one that yielded: (1) the maximum difference between the predicted hit and FA rate among the most separable pair of signal and noise distributions, and (2) the predicted bias in the control condition closest to the behavioral bias $(c \approx 1)$. For Protocol 2 , the decision criterion was fixed across all contrasts for each cell as the mean of the responses to the lowest contrast distractor $\left(0^{\circ}-30 \%\right)$ and the highest contrast target $\left(22.5^{\circ}-70 \%\right)$. For Protocol 3 , the decision criterion was fixed across all ISIs for each cell as the mean of the responses to the most adapted distractor $\left(0^{\circ}-250 \mathrm{~ms}\right.$ ISI) and the most recovered target $\left(22.5^{\circ}-\right.$ $750 \mathrm{~ms}$ ISI). Thus, hit rate or FA rate across all conditions (either contrasts, ISIs, or V1 suppression/excitation) was calculated as percentage of trials of the target or distractor responses that is higher than the decision criterion, respectively.

The predicted hit and FA rate were used to calculate neuronal $d^{\prime}$ and $c$ using the same equations as were used for the behavioral data. To avoid confounds of directionality (because an increase in a positive $d^{\prime}$ and a decrease in a negative $d^{\prime}$ are both increases in sensitivity), only cells that had a positive $d^{\prime}$ in the control condition (V1 suppression: 47/70; V1 excitation: 45/83), or across contrasts (19/92) and ISIs (21/74 cells) were included.

"Nonparametric" measures of sensitivity $\left(A^{\prime}\right.$; Pollack and Norman, 1964) and bias ( $B^{\prime \prime}$; Hodos, 1970; Grier, 1971) were calculated as follows:

$$
\begin{gathered}
A^{\prime}=\frac{1}{2}+\frac{(H R-F A R)(1+H R-F A R)}{4 H R(1-F A R)}, \\
B^{\prime \prime}=\frac{H R(1-H R)-F A R(1-F A R)}{H R(1-H R)+F A R(1-F A R)} .
\end{gathered}
$$

\section{Experimental design and statistical analysis}

All behavioral and neuronal data were tested for normality using a Lilliefors test. Whereas behavioral measures were normally distributed, electrophysiological measures of spike rates were not. Therefore, behavioral data were compared with either a $t$ test or ANOVA with post hoc Tukey HSD test for datasets with two and multiple groups, respectively. However, for the neuronal activity we used only nonparametric tests (Wilcoxon signed rank test and Friedman test with post hoc Tukey HSD test to compare two and multiple groups, respectively). Sample sizes were not predetermined by statistical methods, but are similar to other studies. The numbers of cells, animals, or experiments were provided in the corresponding text, figures, and figure legends. All error values in the text are SEM unless otherwise specified. Data collection and analysis were not performed blind to experimental conditions, but all visual presentation conditions in extracellular recording and behavior experiments are randomized.

Data and code availability

All relevant data and code are available from the corresponding author upon reasonable request.

\section{Results}

To explore whether purely sensory changes can affect measured bias in perceptual decision-making, we designed an orientation discrimination task in which we could (1) measure hit and FA rate to calculate bias and sensitivity, and (2) control the neuronal responses to both targets and distractors (Fig. 1B; Jin et al., 2019). In this task, a head-fixed mouse must press a lever to initiate trials and release it to report a target orientation. Each trial began with the repeated presentation of at least two (and up to nine) isooriented gratings ["distractors": $0^{\circ}$ (vertical) or $45^{\circ}$ (counterclockwise of vertical), $100 \mathrm{~ms}$ duration] followed by a grating of a different orientation from the distractor ("target", range: 9-90 counter-clockwise from the distractor, $100 \mathrm{~ms}$ duration). Within a trial, each stimulus presentation was separated from the preceding presentation by a mean-luminance ISI to eliminate rotation artifacts when transitioning from distractor to target orientations. If the mouse released the lever within a window (200-550 ms; Fig. 1D; details in Materials and Methods) following the onset of the target stimulus, it was considered a hit; if the mouse released the lever within the same window following a distractor stimulus, it was considered a FA. Thus, we could use these behavioral measures to calculate sensitivity and bias using SDT (Green and Swets, 1966).

In addition to being appropriate for making measurements of SDT, this task has a couple of additional advantages. First, the mice performed the task at a high level of proficiency with low lapse rates (measured as 1-hit rate for $90^{\circ}$ targets, $0.053 \pm 0.008$; range $0.003-0.107 ; n=14$ mice $)$, FA rates $(0.048 \pm 0.004$; range $0.032-0.098 ; n=14$ mice), and threshold for orientation discrimination $\left(25.2^{\circ} \pm 1.3^{\circ}\right.$; range $14.2^{\circ}-32.0^{\circ} ; n=14$ mice $)$. Thus, there are minimal concerns about changes in motivational state or arousal that could influence our measures of bias. Second, our previous work has determined how neuronal activity in V1 is used to perform this task: the downstream decoder simply sums V1 spike rates, with higher weight given to neurons that prefer targets while ignoring those that prefer the distractor (Fig. 1C; Jin et al., 2019). Therefore, the decision variables and decision criterion can be approximated in units of firing rate and neuronal responses to targets and distractors can be regarded as signal and noise distributions respectively. Thus, we can use a variety of approaches to alter V1 excitability to coincidently change signal and noise distributions and determine whether these changes in sensory encoding can induce changes in measured bias.

\section{Direct suppression and activation of V1 bidirectionally alters behavioral measures of bias}

To directly test the contribution of sensory encoding in V1 to measures of bias, we optogenetically manipulated the firing rates (FRs) of V1 neurons. We virally or genetically expressed excitatory opsins (ChR2 or Chronos) in either inhibitory or excitatory neurons using transgenic mouse lines (PV::Cre or VGAT-ChR2 and EMX1::Cre). Thus, using these different mouse lines, we can use blue light to either activate inhibitory interneurons to suppress V1 or activate excitatory neurons to excite V1. We applied these optogenetic tools to suppress or excite V1 neurons specifically during presentation of targets or distractors either during performance of the orientation discrimination task (Fig. $1 B, E$ ) or in passively viewing mice (Fig. 2). Notably, the light powers used did not induce significant changes in the lapse rate (V1 suppression vs Control: $0.10 \pm 0.02$ vs $0.08 \pm 0.01 ; p=0.13, n=4$ mice, paired $t$ test; V1 excitation vs Control: $0.06 \pm 0.03$ vs $0.12 \pm 0.06$; $p=0.18, n=4$ mice, paired $t$ test), suggesting similar levels of arousal across conditions. Although orientation discrimination was tested with targets of multiple orientations in each mouse (ranging from 9 to $90^{\circ}$; Fig. $1 E$ ), for simplicity we focused our analysis of behavior and neuronal activity on distractors $\left(0^{\circ}\right)$ and targets near threshold $\left(22.5^{\circ}\right)$.

Extracellular recordings from V1 neurons (Fig. 2A-C) revealed that optogenetic activation of inhibitory neurons significantly reduced neuronal responses to both targets near the animals' discrimination threshold and distractors (FR changes by V1 suppression: $22.5^{\circ}$ : $-6.6 \pm 1.2 \mathrm{~Hz}, p<10^{-9} ; 0^{\circ}$ : $-5.5 \pm 1.2$ $\mathrm{Hz}, p<10^{-10} ; n=70$ cells; Wilcoxon signed rank test; Fig. $2 D, E)$, whereas activation of excitatory neurons increased visually driven responses (FR changes by V1 excitation: $22.5^{\circ}: 2.6 \pm$ $0.4 \mathrm{~Hz}, p<10^{-8} ; 0^{\circ}: 2.5 \pm 0.3 \mathrm{~Hz}, p<10^{-11} ; n=83$ cells; Wilcoxon signed rank test). Importantly, these effects of optogenetic manipulation were largely temporally selective, as we saw little to no effect on stimuli $\left(\operatorname{Stim}_{\mathrm{N}}\right)$ for which the preceding stimulus $\left(\right.$ Stim $\left._{\mathrm{N}-1}\right)$ was optogenetically manipulated (FR changes by V1 suppression: $22.5^{\circ}: p=0.02 ; 0^{\circ}: p=0.50$; FR 
A
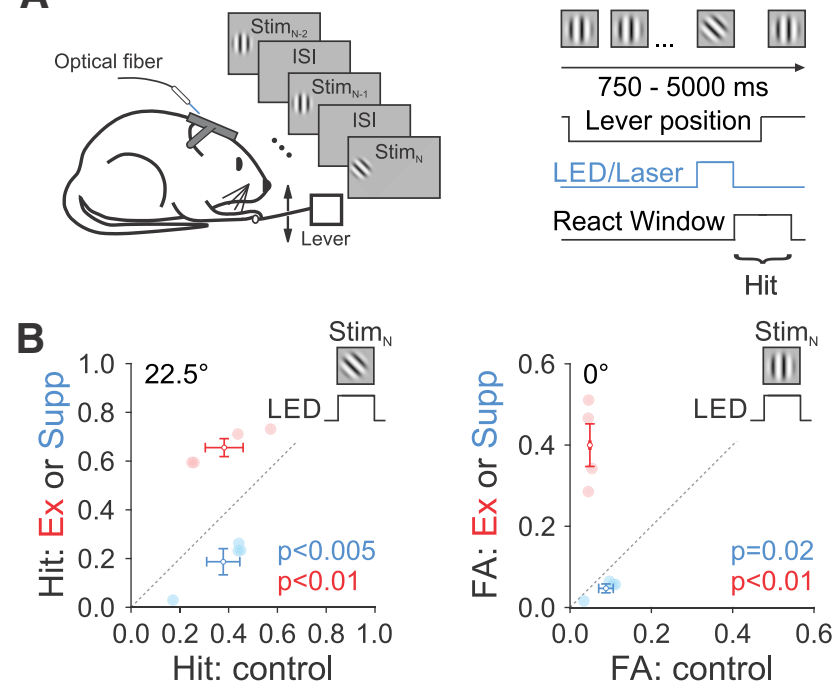

C
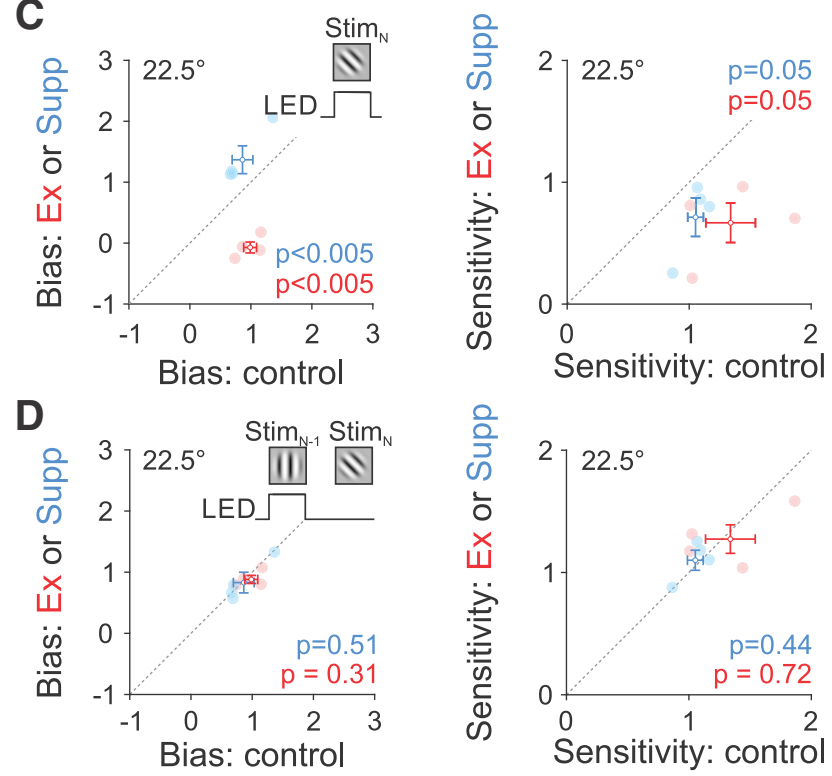

Figure 3. Suppressing or exciting V1 increases or decreases behavioral bias. A, Schematic of behavior setup and trial progression. Stim $_{N}$ is the stimulus that the animal responded to by releasing the lever and $\mathrm{Stim}_{\mathrm{N}-1}$ is the stimulus preceding $\mathrm{Stim}_{\mathrm{N}}$. V1 suppression (blue) and excitation (red) is achieved via optogenetically driving PV + or VGAT + neurons and Emx1+ neurons, respectively. $\boldsymbol{B}$, Comparison of the hit $\left(22.5^{\circ}\right.$; left) and $\mathrm{FA}$ rate $\left(0^{\circ}\right.$; right) between control and V1 suppression (blue; $n=4$ mice) or excitation (red; $n=4$ mice) on Stim ${ }_{\mathrm{N}}$. Filled circles are individual mice and error bars are SEM across mice with white dots in the center showing the mean of the population. $\boldsymbol{C}$, Same as $\boldsymbol{B}$, for bias (left) and sensitivity (right) at $22.5^{\circ}$. $D$, Same as $C$, for $\mathrm{V} 1$ suppression or excitation on Stim $_{\mathrm{N}-1}$.

changes by V1 excitation: $22.5^{\circ}: p=0.58 ; 0^{\circ}: p=0.79$; Wilcoxon signed rank test; Fig. $2 F$ ).

Because we think that the decision variable is closely related the total firing rate in V1 (Jin et al., 2019), we expect that the reduction in firing rates because of activation of inhibitory interneurons will decrease the decision variable and make it less likely to cross the decision threshold. Consistent with this hypothesis, we found that optogenetic suppression of activity in $\mathrm{V} 1$ reduced behavioral hit rate $\left(22.5^{\circ}\right.$ target: V1 suppression vs Control: $p<0.005 ; n=4$ mice; paired $t$ test; Figs. $1 E, 3 B)$ and FA rate (V1 suppression vs Control: $p=0.02 ; n=4$ mice; paired $t$ test). These associated changes in both hit and FA rate resulted in changes in bias $(c)$ measured by SDT. Indeed, we found a signif- icant increase in measured bias ( $c$ for $22.5^{\circ}$ target: $\mathrm{V} 1$ suppression vs Control: $p<0.005$; paired $t$ test; Fig. $3 C$ ) and a slight decrease in sensitivity ( $d^{\prime}$ for $22.5^{\circ}$ target: $\mathrm{V} 1$ suppression vs Control: $p=$ 0.05 ; paired $t$ test). Conversely, optogenetic excitation of $\mathrm{V} 1$ increased behavioral hit rate $\left(22.5^{\circ}\right.$ target: $\mathrm{V} 1$ excitation vs Control: $p<0.01 ; n=4$ mice; paired $t$ test; Figs. $1 E, 3 B)$ and FA rate (V1 excitation vs Control: $p<0.01 ; n=4$ mice; paired $t$ test), resulting in a decrease in measured bias ( $c$ for $22.5^{\circ}$ target: V1 excitation vs Control: $p<0.005$; paired $t$ test; Fig. $3 C$ ) and a slight decrease in sensitivity ( $d^{\prime}$ for $22.5^{\circ}$ target: V1 excitation vs Control: $p=$ 0.05 ; paired $t$ test). As with the neuronal data, the effects of optogenetic manipulation were temporally selective: optogenetic manipulation of activity during a stimulus had no effect on bias or sensitivity on the subsequent stimulus (V1 suppression, $c: p=$ $0.51 ; d^{\prime}: p=0.44 ; \mathrm{V} 1$ excitation, $c: p=0.31 ; d^{\prime}: p=0.72$; paired $t$ test; Fig. 3D).

Notably, there are cognitive contributions to bias that cause fluctuations over the duration of a trial or session. For instance, because the hazard function for target appearance is not flat, the expectation of a target increases with trial duration (Kang and Maunsell, 2012; Jin et al., 2019), and this will decrease bias. Indeed, we found that thresholds on short trials ( $\leq 2$ s) were slightly, though not significantly, higher than on long trials $(\geq 3 \mathrm{~s}$; long vs short: $24.9^{\circ} \pm 2.2^{\circ}$ vs $26.9^{\circ} \pm 1.7^{\circ} ; p=0.37 ; n=8$ mice; paired $t$ test) and FA rates on short trials were slightly, but not significantly, lower than on long trials (long vs short: $0.08 \pm 0.02$ vs $0.06 \pm 0.01 ; p=0.27 ; n=8$ mice; paired $t$ test). However, the effects of optogenetic manipulations in V1 on bias were not dependent on trial length (interaction between V1 excitability and trial length, $\mathrm{V} 1$ suppression: $p=0.71, \mathrm{DF}=1$; V1 excitation: $p=$ $0.79, \mathrm{DF}=1$; two-way ANOVA), or number of distractor presentations (interaction between $\mathrm{V} 1$ excitability and distractor numbers (2-4 vs $6-8$ distractors: V1 suppression: $p=0.76$, $\mathrm{DF}=1$; V1 excitation: $p=0.71, \mathrm{DF}=1$; two-way ANOVA). Thus, the observed effects of $\mathrm{V} 1$ excitability on bias were robust to fluctuations in bias over the duration of a trial.

\section{Direct suppression and activation of V1 bidirectionally alters neuronal measures of bias}

To test whether the changes in sensory encoding in V1 can qualitatively account for the changes in behavioral bias, we predicted neuronal bias by using neuronal activity in V1 recorded during the passively viewing condition. These recordings were performed in non-behaving mice to avoid the potential contribution of feedback from decision-making areas. We used these data to determine whether the predicted neuronal bias changed in the same direction as observed behaviorally. We first constructed the signal and noise distributions for each cell as its response to $22.5^{\circ}$ targets and $0^{\circ}$ distractors, respectively, and then applied a decision criterion across control and optogenetic manipulation conditions to predict hit and FA rate. The decision criterion was fixed across optogenetic conditions and was chosen as the mean of each cell's responses to the maximum and minimum response conditions to avoid conditions that resulted in ceiling or floor effects on the hit and FA rates (Fig. 4A). For V1 suppression, we chose the criterion as the mean between the average responses to optogenetically suppressed distractors $\left(0^{\circ}\right)$ and control targets $\left(22.5^{\circ}\right)$; for $\mathrm{V} 1$ excitation, we chose the mean between the average responses to control distractors $\left(0^{\circ}\right)$ and optogenetically excited targets $\left(22.5^{\circ}\right)$. This criterion was then applied to the neuronal response distributions such that responses larger than the criterion were considered hits or FAs, whereas responses smaller than the criterion were considered misses or correct rejects. These 
A

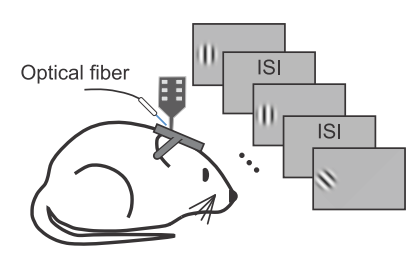

B

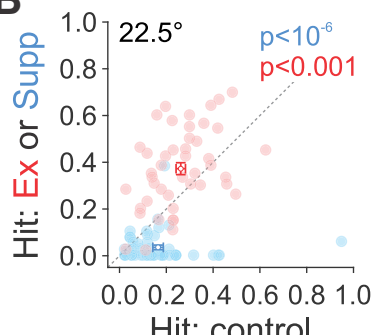

C

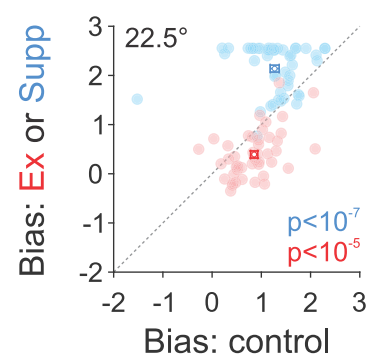

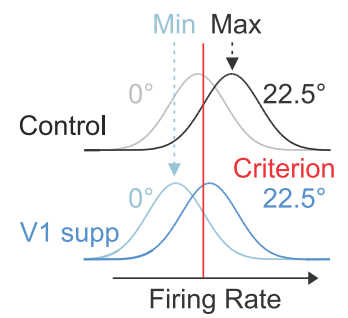
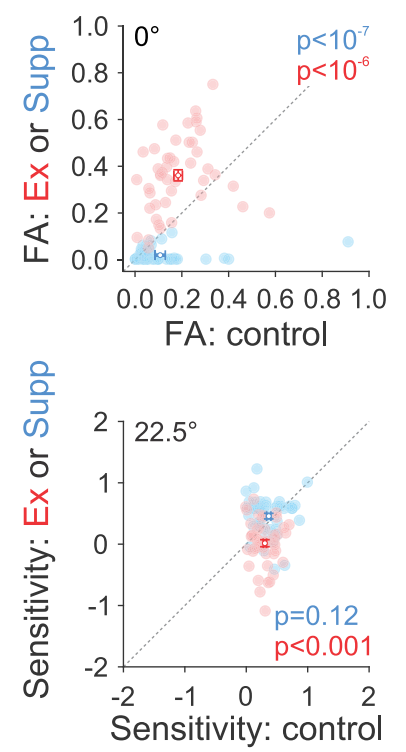

Figure 4. Suppressing or exciting V1 increases or decreases neuronal bias. $\boldsymbol{A}$, Left, Schematic of extracellular recording setup. Right, For V1 suppression, the criterion (solid red line) is set as the mean of each cell's responses to the maximum and minimum response conditions. In the case of optogenetic suppression, this is the mean of the $22.5^{\circ}$ targets in the control condition (black) and the suppressed $0^{\circ}$ distractors (light blue). $\boldsymbol{B}$, Predicted hit (22.5 $5^{\circ}$; left) and FA rate $\left(0^{\circ}\right.$; right) from neuronal responses using a fixed criterion for each cell (see Materials and Methods). Error is SEM across cells (V1 suppression-blue: $n=47$ cells, 3 mice; V1 excitation-red, $n=45$ cells, 3 mice). C, Predicted bias (left) and sensitivity (right) using the predicted hit and FA rate in $\boldsymbol{B}$.

were then used to calculate "predicted" hit and FA rates. Because the decoder in this task largely monitors target preferring cells while ignoring distractor preferring cells (Fig. 1C; Jin et al., 2019), we only focused on cells that had a positive $d^{\prime}$ in the control condition. In addition, this selection criterion aids in the interpretation of changes in $d^{\prime}$ : in neurons with positive $d^{\prime}$, increases in $d^{\prime}$ reflect increases in discriminability, whereas the opposite is true for neurons with negative $d^{\prime}$.

Suppressing neuronal activity in V1 decreased the predicted hit rate $\left(22.5^{\circ}\right.$ target: V1 suppression vs Control: $p<10^{-6} ; n=$ 47 cells; Wilcoxon signed rank test; Fig. $4 B$ ) and FA rate (V1 suppression vs Control: $p<10^{-7} ; n=47$ cells; Wilcoxon signed rank test) leading to an increase in measured bias ( $c$ for $22.5^{\circ}$ target: V1 suppression vs Control: $p<10^{-7}$; Wilcoxon signed rank test; Fig. $4 C$ ) without significantly changing the sensitivity ( $d^{\prime}$ for $22.5^{\circ}$ target: V1 suppression vs Control: $p=0.12$; Wilcoxon signed rank test). Conversely, increasing neuronal activity in V1 increased predicted hit $\left(22.5^{\circ}\right.$ target: V1 excitation vs Control: $p<0.001 ; n=45$ cells; Wilcoxon signed rank test; Fig. $4 B$ ) and FA rate (V1 excitation vs Control: $p<10^{-6} ; n=45$ cells; Wilcoxon signed rank test), resulting in a decrease in measured bias ( $c$ for $22.5^{\circ}$ target: V1 excitation vs Control: $p<10^{-5}$; Wilcoxon signed rank test; Fig. $4 C$ ) and sensitivity ( $d^{\prime}$ for $22.5^{\circ}$ tar- get: V1 excitation vs Control: $p<0.001$; Wilcoxon signed rank test).

These effects on bias were not due to our neuronal selection criterion. If we only focused on neurons with $d^{\prime}<0$ (distractor preferring neurons), the effects of V1 excitability on measured bias were in the same direction ( $c$ for $22.5^{\circ}$ target: $V 1$ suppression vs Control: $1.95 \pm 0.16$ vs $0.02 \pm 0.38 ; p<10^{-4} ; n=23$ cells; V1 excitation vs Control: $0.43 \pm 0.08$ vs $0.68 \pm 0.19 ; p=0.03 ; n=38$ cells; Wilcoxon signed rank test). Nor were the effects on bias because of our chosen decision criterion. If instead of choosing the mean firing rate, we chose a decision criterion that maximized the difference between hit and FA rate, the effects of V1 excitability on neuronal bias remained in the same direction $\left(c\right.$ for $22.5^{\circ}$ target: V1 suppression vs Control: $1.82 \pm 0.13$ vs $0.08 \pm 0.26, p<$ $10^{-8} ; n=47$ cells; V1 excitation vs Control: $0.41 \pm 0.18$ vs $0.98 \pm$ $0.15, p<10^{-7} ; n=45$ cells; Wilcoxon signed rank test). Alternatively, if we chose a decision criterion that matched the bias measured in control behavioral conditions $(c \approx 1$; notably, this bias is conservative, likely because of the high ratio of distractor to target presentations), we still observed similar effects of manipulating V1 activity on bias ( $c$ for $22.5^{\circ}$ target: V1 suppression vs Control: $2.14 \pm 0.07$ vs $1.38 \pm 0.06, p<10^{-7}$; V1 excitation vs Control: $0.78 \pm 0.09$ vs $1.16 \pm 0.07, p<0.001)$.

SDT measures have underlying assumption that the decision variable distributions are standard normal. Although we are unable to preclude that the true decision variable distributions are not normal, the neuronal responses were not. To determine whether the normality assumption is necessary for our main conclusion about the directionality of changes in bias by V1 manipulation, we also measured "nonparametric" equivalences of sensitivity ( $A^{\prime}$; Pollack and Norman, 1964) and bias ( $B^{\prime \prime}$; Hodos, 1970; Grier, 1971). Similar to parametric measures, we found that V1 suppression induced an increase in $B^{\prime \prime}$ (for $22.5^{\circ}$ target: V1 suppression vs Control: $0.47 \pm 0.05$ vs $0.27 \pm 0.03, p<10^{-3} ; n=$ 47 cells; Wilcoxon signed rank test), whereas V1 excitation induced a decrease in $B^{\prime \prime}$ (V1 excitation vs Control: $-0.02 \pm 0.02$ vs $0.16 \pm 0.02, p<10^{-6} ; n=45$ cells; Wilcoxon signed rank test). We also observed a slight increase in $A^{\prime}$ when suppressing V1 $\left(A^{\prime}\right.$ for $22.5^{\circ}$ target: $\mathrm{V} 1$ suppression vs Control: $0.64 \pm 0.02$ vs $0.63 \pm$ $0.01 ; p=0.04 ; n=47$ cells) and a decrease in $A^{\prime}$ when exciting V1 (V1 excitation vs Control: $0.47 \pm 0.03$ vs $0.61 \pm 0.01 ; p<10^{-4}$; $n=47$ cells). This suggests that our results are robust to the assumed distributions of the decision variables.

Together, our electrophysiology data show that manipulating excitability of neurons in V1 is sufficient to alter bias even in the absence of a flexible decision criterion and decision-related feedback signals. Moreover, the neuronal and behavioral changes in bias are in the same direction, suggesting that the changes in sensory encoding could be responsible for the changes in behavioral bias.

\section{V1 suppression also increases behavioral bias in a contrast detection task}

The interpretation of the effects of optogenetically manipulating firing rates during the orientation discrimination task depends in part on the presumed decision variable: namely, that the downstream perceptual choice circuit is monitoring the firing rates of the neurons in the visual cortex. This is also likely the decision variable used for contrast detection. To demonstrate that the effects of V1 excitability on measured bias is a general phenomenon across behavioral tasks with similar decoding strategy, we tested whether V1 suppression also increases bias in a contrast detection task (Fig. 5). In this task, mice pressed the lever to 

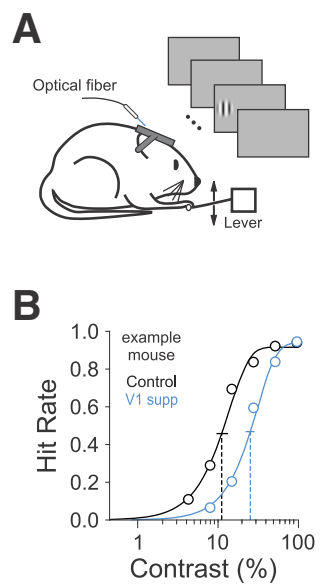

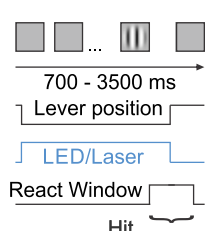

Hit

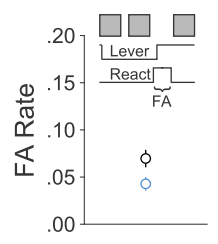

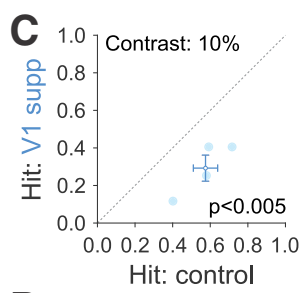

D

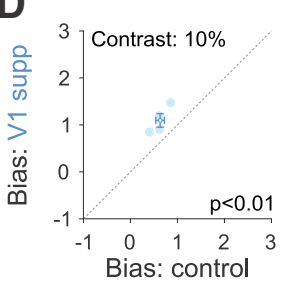

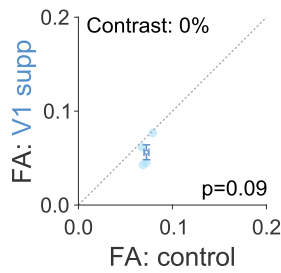

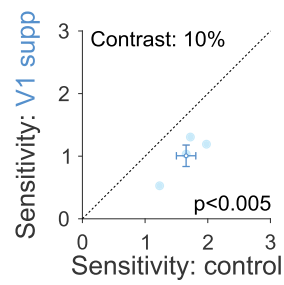

Figure 5. Suppressing V1 increases behavioral bias in a contrast detection task. $A$, Schematic of behavior setup and trial progression for a contrast detection task. Blue light is turned on during the entire length of a trial. V1 suppression (blue) is achieved via optogenetically driving PV + or VGAT + neurons. $\boldsymbol{B}$, Hit rate (left) and FA rate (right) for control (black) and V1 suppression (blue) for one example mouse. Hit rates are fitted with a Weibull function; vertical dotted lines are threshold, error is $95 \%$ confidence interval. C, Comparison of the hit (10\% contrast; left) and FA rate ( $0 \%$ contrast; right) between control and V1 suppression (blue; $n=4$ mice). Filled circles are individual mice and error bars are SEM across mice with white dots in the center showing the mean of the population. $\boldsymbol{D}$, Same as $\boldsymbol{C}$, for bias (left) and sensitivity (right) at $10 \%$ contrast.

initiate each trial and release it when a grating (contrast range: $4-100 \%$ ) appeared on the gray screen (Fig. $5 A, B$ ). Like the orientation discrimination task, the mice had low lapse rates (measured as 1-hit rate for $100 \%$ contrast: $0.065 \pm 0.012$; range $0.038-$ $0.086 ; n=4$ mice $),$ FA rates $(0.072 \pm 0.003$; range $0.068-0.080$; $n=4$ mice), and threshold for contrast detection $(7.7 \% \pm 1.2 \%$; range $5.8 \%-11.1 \% ; n=4$ mice). V1 suppression was achieved by optogenetically activating inhibitory interneurons [in PV::Cre $(n=2)$ and VGAT-ChR2 $(n=2)$ mice]. The light power was also titrated so that there was no difference between lapse rate across conditions (V1 suppression vs Control: $0.06 \pm 0.004$ vs $0.06 \pm$ $0.01 ; p=0.83, n=4$ mice, paired $t$ test). As in the orientation discrimination task, we found that V1 suppression significantly decreased hit rate (10\% contrast: V1 suppression vs Control: $p<$ 0.005; $n=4$ mice; paired $t$ test; Fig. $5 C$ ) and slightly, but not significantly, reduced FA rate (V1 suppression vs Control: $p=$ $0.09 ; n=4$ mice; paired $t$ test), resulting in a significant increase in measured bias ( $c$ for $10 \%$ contrast: V1 suppression vs Control: $p<0.01$; paired $t$ test; Fig. $5 D)$ and decrease in sensitivity $\left(d^{\prime}\right.$ for 10\% contrast: V1 suppression vs Control: $p<0.005$; paired $t$ test). Thus, manipulation of V1 activity is sufficient to alter bias in multiple perceptual tasks with similar decoding strategies.

\section{Manipulation of stimulus contrast affects measures of bias}

Optogenetic tools allow for the direct manipulation of firing rates, however any manipulation that coincidently increases or decreases firing rates in response to targets and distractors is predicted to impact measures of bias. For instance, neurons in V1 usually have monotonic contrast-response functions (Ohzawa et al., 1982), and therefore decreasing stimulus contrast should decrease firing rates in response to both targets and distractors, shifting the optimal criterion to lower stimulus values. Thus, we modified our orientation discrimination task to vary stimulus contrast (30, 50 and 70\%) on a presentation-by-presentation basis (Fig. 6A). We chose this range of contrast (30-70\%) to ensure that there was no significant difference between the lapse rates across contrast conditions (measured as 1-hit rate for $90^{\circ}$ targets, 70 vs $30 \%$ : $0.06 \pm 0.02$ vs $0.10 \pm 0.02 ; p=0.32, \mathrm{DF}=2 ; n=5$ mice, one-way ANOVA). Extracellular recordings confirm that manipulation of contrast significantly affected firing rates in response to both targets $\left[22.5^{\circ}\right.$ : FR changes from 70 to $30 \%$ :
$-4.5 \pm 0.7 \mathrm{~Hz}, p<10^{-7} ; n=92$ cells; $\mathrm{DF}=2$; Friedman test $\left(p<10^{-8}\right)$ with post hoc Tukey HSD test; Fig. $\left.6 \mathrm{~B}\right]$ and distractors $\left[0^{\circ}\right.$ : FR changes from 70 to $30 \%:-4.2 \pm 0.5 \mathrm{~Hz}, p<10^{-9}$; $n=92$ cells; Friedman test $\left(p<10^{-25}\right)$ with post hoc Tukey HSD test].

Consistent with lower stimulus contrast driving lower firing rates, we found that decreasing stimulus contrast significantly reduced the animal's hit rate $\left[22.5^{\circ}\right.$ target- one-way ANOVA $(p=0.05 ; \mathrm{DF}=2)$ with post hoc Tukey HSD test for 70 vs $30 \%$ : $p=0.04 ; n=5$ mice; Fig. $6 C$ ] and FA rate [one-way ANOVA $\left(p<10^{-3}\right)$ with post hoc Tukey HSD test for 70 vs 30\%: $p<$ $\left.10^{-3}\right]$. These changes in hit and FA rate resulted in a significant increase in bias ( $c$ for $22.5^{\circ}$ target: one-way ANOVA $(p<0.005)$ with post hoc Tukey HSD test for 70 vs $30 \%$ : $p<0.005$; Fig. $6 D$ ) without a significant change in sensitivity $\left(d^{\prime}\right.$ for $22.5^{\circ}$ target: one-way ANOVA; $p=0.81)$. Notably, we observed a larger effect on bias when comparing low (30\%) vs high (70\%) contrast, than when comparing low (30\%) vs intermediate (50\%) contrast (difference in bias: $30-70$ vs $30-50 \%$ : $0.88 \pm 0.10$ vs $0.43 \pm 0.05 ; p<$ 0.005; paired $t$ test). This suggests that the size of the effect on measured bias depends on the size of the effect on sensory encoding.

As with optogenetic manipulation of neuronal activity, the effects of manipulating visual stimulus features on behavior were consistent with the observed effects on V1 activity. Lowering stimulus contrast decreased both the predicted hit rate $\left[22.5^{\circ}\right.$ target: Friedman test $(p<0.01, \mathrm{DF}=2)$ with post hoc Tukey HSD test for 70 vs 30\%: $p=0.02 ; n=19$ cells; Fig. $6 E]$ and FA rate [Friedman test $\left(p<10^{-6}\right)$ with post hoc Tukey HSD test for $70 \mathrm{vs}$ $\left.30 \%: p<10^{-5}\right]$, resulting in an increase in measured bias [ $c$ for $22.5^{\circ}$ target: Friedman test $\left(p<10^{-3}\right)$ with post hoc Tukey HSD test for 70 vs $30 \%$ : $p<10^{-3}$; Fig. $6 F$ ] without significantly changing the predicted sensitivity ( $d^{\prime}$ for $22.5^{\circ}$ target: Friedman test, $p=0.08$ ). Thus, changes in the quality of sensory encoding through variation of visual stimulus properties can affect behavioral and neuronal measures of bias.

\section{Manipulation of adaptation state affects measures of bias}

Varying stimulus contrast revealed that stimulus manipulations of sensory encoding can affect measured bias. To demonstrate the ubiquity of this phenomenon, we manipulated a different prop- 
A
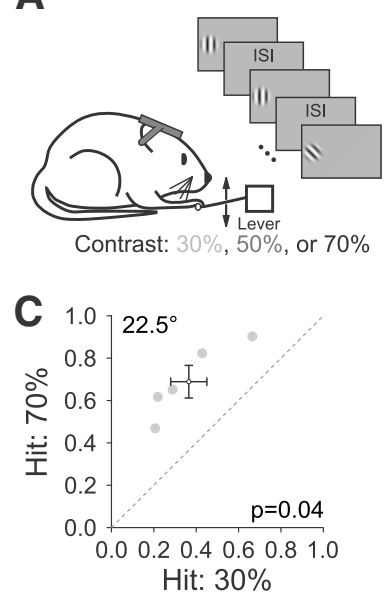

D

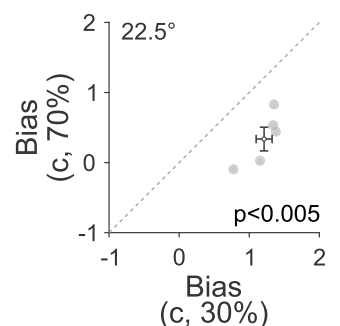

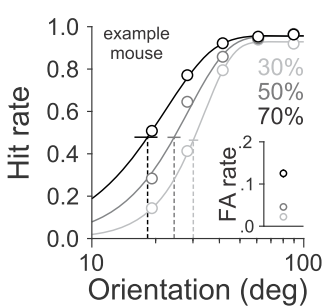
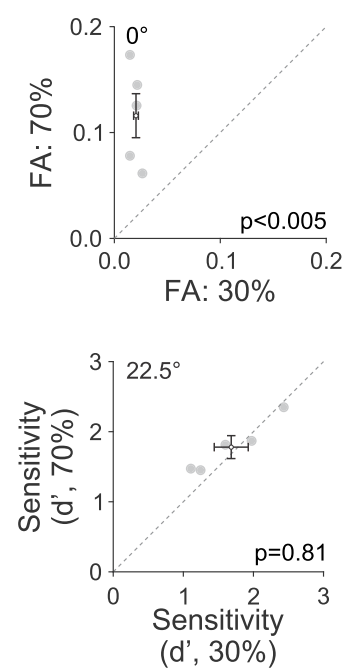

B
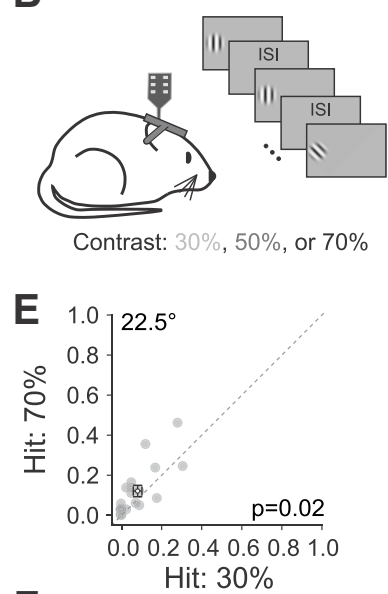

$\mathbf{F}$

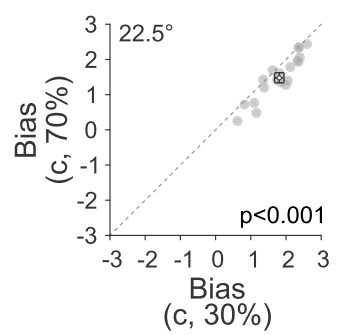

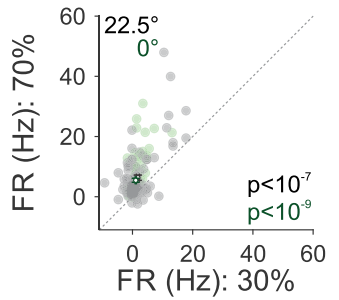
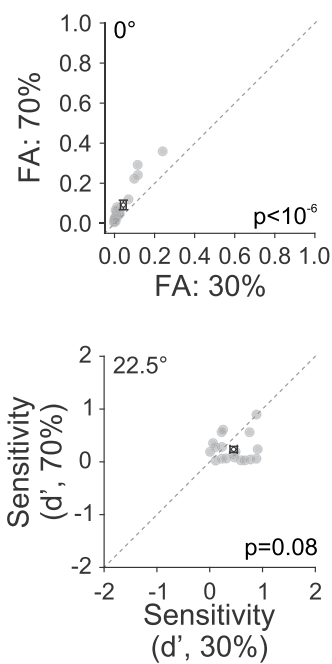

Figure 6. Decreasing stimulus contrast decreases both hit and FA rate and increases behavioral and neuronal bias in the orientation discrimination task. $\boldsymbol{A}$, Left, Schematic of behavioral setup. Stimulus contrast is varied [30\% (light gray), $50 \%$ (dark gray), or 70\% (black)] on each stimulus presentation. Right, Hit rate and FA rate (inset) for each contrast for an example mouse. Hit rates are fit with a Weibull function; vertical dotted lines are threshold, error is $95 \%$ confidence interval. $\boldsymbol{B}$, Left, Schematic of extracellular recording setup. Right, Comparison of neuronal responses (FR, in hertz) to the $22.5^{\circ}$ target (black) and $0^{\circ}$ distractor (green) between 30 and $70 \%$ contrast. Filled circles are individual cells and error bars are SEM across cells with white dots in the center showing the mean of the population ( $n=92$ cells, 4 mice). C, Comparison of behavioral hit (left; $22.5^{\circ}$ target) and FA rate (right; $0^{\circ}$ distractor) between two contrasts (70 vs $30 \%$ ). Filled circles are individual mice and error bars are SEM across mice with white dots in the center showing the mean of the population $(n=5)$. D, Same as $\boldsymbol{C}$, for bias (left) and sensitivity (right) at $22.5^{\circ}$. $\boldsymbol{E}, \boldsymbol{F}$, Same as $\boldsymbol{C}$ and $\boldsymbol{D}$, for predicted $(\boldsymbol{E})$ hit and FA rate and $(\boldsymbol{F})$ bias and sensitivity from the neuronal data ( $n=19$ cells, 4 mice).

erty of the task design that affects sensory encoding: ISI $(250,500$, and $750 \mathrm{~ms}$; Fig. 7A). Varying the ISI, like varying contrast, alters the strength of sensory responses, where shorter ISIs drive suppressive adaptation and lower firing rates (Clifford et al., 2007; Jin et al., 2019). Indeed, extracellular recordings revealed that adaptation significantly decreased the neuronal responses to distractors [0 $: 250 \mathrm{~ms}: 6.3 \pm 1.1 \mathrm{~Hz} ; 500 \mathrm{~ms}: 8.7 \pm 1.5 \mathrm{~Hz} ; 750 \mathrm{~ms}$ : $10.2 \pm 1.6 \mathrm{~Hz}$; Friedman test $\left(p<10^{-9}, \mathrm{DF}=2\right)$ with post hoc Tukey HSD test for FR changes from 750 to $250 \mathrm{~ms}$ ISI: $p<10^{-8}$; $n=74$ cells; Fig. $7 B$ ], whereas slightly, but not significantly, decreasing responses to targets $\left(22.5^{\circ}: 250 \mathrm{~ms}: 9.3 \pm 1.4 \mathrm{~Hz} ; 500 \mathrm{~ms}\right.$ : $10.4 \pm 1.7 \mathrm{~Hz}$; 750:11.7 $\pm 1.8 \mathrm{~Hz}$; Friedman test, $p=0.17$ ). Although there was an asymmetric effect of ISI on targets and distractors (consistent with the stimulus-specific effects of adaptation; Müller et al., 1999; Dragoi et al., 2000), the net effect of adaptation is to reduce firing rates and this should decrease the optimal criterion and therefore increase bias.

Consistent with this prediction, decreasing the ISI decreased both hit rate $\left[22.5^{\circ}\right.$ target: one-way ANOVA $(p=0.02 ; \mathrm{DF}=2)$ with post hoc Tukey HSD test for 750 vs $250 \mathrm{~ms}: p=0.01 ; n=11$ mice; Fig. $7 C$ ] and FA rate [one-way ANOVA $\left(p<10^{-8}\right)$ with post hoc Tukey HSD test for 750 vs $250 \mathrm{~ms}: p<10^{-8}$ ]. The decrease in both hit and FA rate supported an increase in measured bias [ $c$ for $22.5^{\circ}$ target: one-way ANOVA $\left(p<10^{-3}\right)$ with post hoc Tukey HSD test for 750 vs $250 \mathrm{~ms}: p<10^{-4}$; Fig. $7 D$ ], without a coincident change in sensitivity ( $d^{\prime}$ for $22.5^{\circ}$ target: one-way ANOVA, $p=0.85$ ). Importantly, the range of ISIs selected here did not affect lapse rate such that there was minimal concern of different arousal states across ISI conditions (750 vs 250 ms: $0.05 \pm 0.01$ vs $0.05 \pm 0.01 ; p=0.97$; $\mathrm{DF}=2$; one-way ANOVA). Similar to the contrast manipulation, the size of the effect on bias depended on the size of the effect on sensory encoding (difference in bias: $250-750$ vs $500-750 \mathrm{~ms}: 0.50 \pm 0.04$ vs $0.32 \pm 0.02 ; p<10^{-3}$; paired $t$ test).

As with manipulating contrast, the behavioral effects of manipulating ISI were expected from the observed changes in neuronal activity recorded in V1. Using a fixed-decision criterion, the decreased responses to targets and distractors with decreasing ISI significantly decreased the predicted FA rate $\left[0^{\circ}\right.$ : Friedman test $\left(p<10^{-4}, \mathrm{DF}=2\right)$ with post hoc Tukey HSD test for 750 vs 250 ms: $p<10^{-4} ; n=21$ cells; Fig. $\left.7 E\right]$ and slightly, but not significantly, decreased the hit rate $\left(22.5^{\circ}\right.$ target: Friedman test, $p=$ 0.37 ) resulting in an increase in measured bias [ $c$ for $22.5^{\circ}$ target: Friedman test $(p<0.005)$ with post hoc Tukey HSD test for $750 \mathrm{vs}$ $250 \mathrm{~ms}: p<0.005$; Fig. $7 F$ ] without a change in sensitivity $\left(d^{\prime}\right.$ for $22.5^{\circ}$ target: Friedman test, $p=0.26$ ). Thus, the effects of ISI on behavioral bias were consistent with the effects of ISI on sensory encoding. Together, these data demonstrate that both direct optogenetic, and indirect stimulus-dependent, manipulations of sensory encoding affect both behavioral and neuronal measures of bias.

\section{Discussion}

Here we provide both behavioral and neuronal evidence that measures of bias are sensitive to changes in sensory encoding. Directly manipulating neuronal excitability in V1 induced pre- 
A

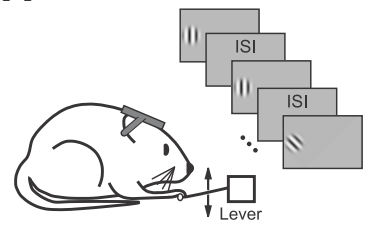

ISI: 250,500 , or $750 \mathrm{~ms}$

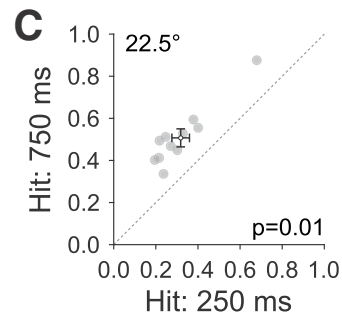

D

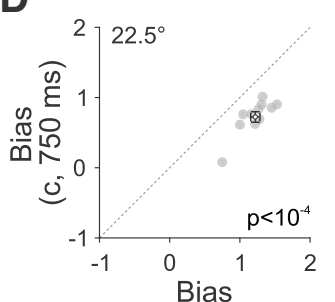

(c, $250 \mathrm{~ms}$ )
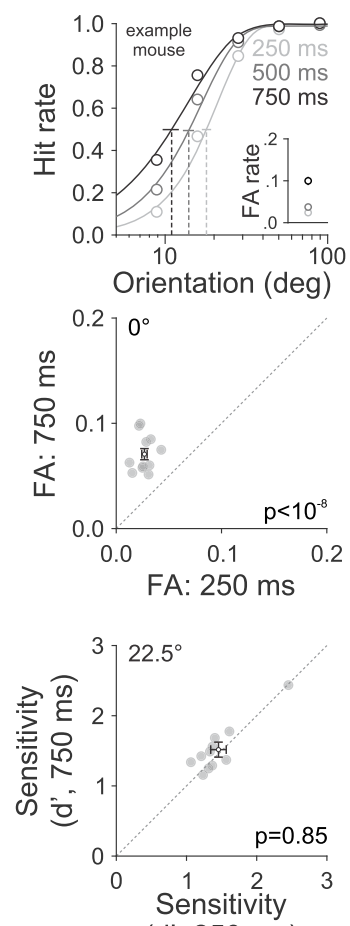

(d', $250 \mathrm{~ms}$ )
B

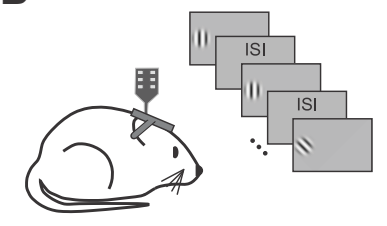

ISI: 250,500 , or $750 \mathrm{~ms}$

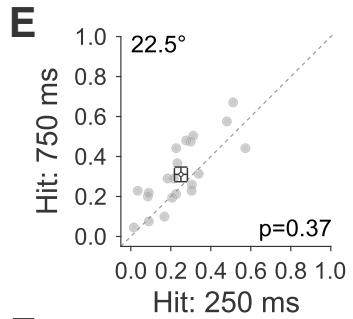

$\mathbf{F}$

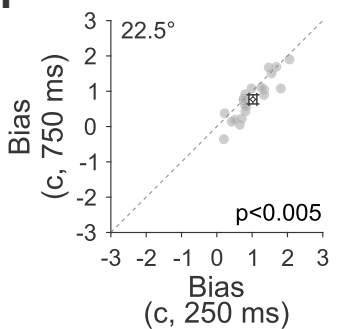

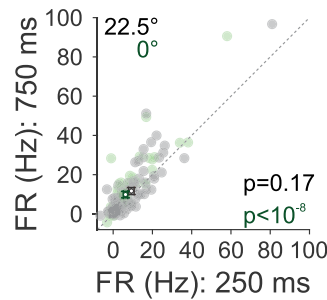
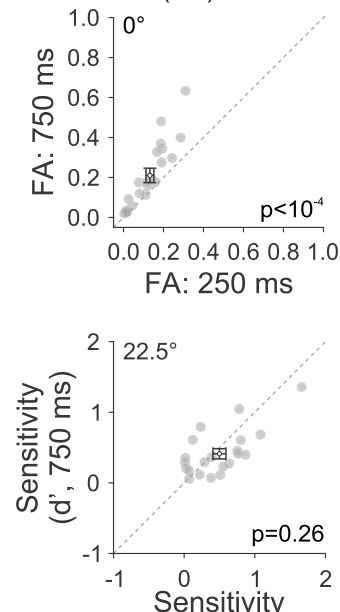

(d', $250 \mathrm{~ms}$ )

Figure 7. Adaptation decreases both hit and FA rate and increases behavioral and neuronal bias in the orientation discrimination task. $\boldsymbol{A}$, Left, Schematic of behavioral setup. ISI is varied [250 ms (light gray), $500 \mathrm{~ms}$ (dark gray) or $750 \mathrm{~ms}$ (black)] on each stimulus presentation. Right, Hit rate and FA rate (inset) for each ISI for an example mouse. Hit rates are fit with a Weibull function; vertical dotted lines are threshold, error is $95 \%$ confidence interval. $\boldsymbol{B}$, Left, Schematic of extracellular recording setup. Right, Comparison of neuronal responses (FR, in hertz) to the $22.5^{\circ}$ target (black), and $0^{\circ}$ distractor (green) after 750 or $250 \mathrm{~ms}$ ISIs. Filled circles are individual cells and error bars are SEM across cells with white dots in the center showing the mean of the population ( $n=74$ cells, 4 mice). $\boldsymbol{C}$, Comparison of behavioral hit (left; $22.5^{\circ}$ target) and FA rate (right; $0^{\circ}$ distractor) between two ISIs ( $750 \mathrm{vs} 250 \mathrm{~ms}$ ). Filled circles are individual mice and error bars are SEM across mice with white dots in the center showing the mean of the population $(n=11)$. $\boldsymbol{D}$, Same as $\boldsymbol{C}$, for bias (left) and sensitivity (right) at $22.5^{\circ}$. $\boldsymbol{E}, \boldsymbol{F}$, Same as $\boldsymbol{C}$ and $\boldsymbol{D}$, for predicted $(\boldsymbol{E})$ hit and FA rate and $(\boldsymbol{F})$ bias and sensitivity from the neuronal data ( $n=21$ cells, 4 mice).

dictable changes in behavioral bias with comparatively little effect on sensitivity in the performance of both an orientation discrimination task and a contrast detection task. Moreover, by varying either stimulus contrast or adaptation state in the orientation discrimination task, we also observed robust changes in bias. Changes in the firing rates of neurons in V1 reflect these same changes in bias, even in the absence of a flexible decision criterion and feedback of decision-making signals. These results clearly demonstrate that changes in bias are not necessarily solely because of cognitive mechanisms, and conversely, that the lack of a change in sensitivity does not preclude effects on sensory encoding.

There have been previous reports that causal manipulations of sensory areas can drive biases in behavioral choices. Electrical stimulation or optogenetic inactivation of direction-selective neurons in the sensory visual area MT can bias the monkey's choice toward or away from the neurons' preferred direction in two-alternative forced choice (2AFC) tasks, suggesting that manipulation of sensory areas can induce perceptual biases (Salzman et al., 1990; Ditterich et al., 2003; Fetsch et al., 2018). However, 2AFC tasks are criterion free and therefore not amenable to bias measured by SDT as in go/no-go tasks. In go/no-go tasks, there have been previous reports that manipulations of areas related to the decision-making process can drive biases in response probability. For instance, unilateral manipulation of superior colliculus in monkeys and the dorsal striatum in mice both alter bias in go/no-go change detection tasks (Herman et al., 2018; Wang et al., 2018). However, whether manipulation of activity in a primary sensory area could reliably and predictably alter perceptual bias as measured with SDT was less clear. Here we demonstrate that activating or inactivating sensory area V1 can decrease or increase bias in a go/no-go orientation discrimination task.

We think it is unlikely that the observed effects on bias in our study are entirely because of changes in the animals' decision criterion because we have carefully limited the contributions of several cognitive factors that might otherwise impact bias. First, all conditions (optogenetic stimulation, ISI, and contrast) were varied on a presentation-by-presentation basis within each trial such that the animal could not predict the upcoming condition. Together with the lack of effect on stimuli immediately following the manipulation, it is unlikely that the mouse could adjust its decision criterion on these short time scales. Second, these manipulations do not significantly affect lapse rate across conditions suggesting that levels of arousal and engagement are constant. Finally, although animals might be more liberal as trial length or the number of distractors increases in a trial, the effects of optogenetic manipulations on bias remained the same regardless of whether the stimuli appeared either early or late in a trial or how many distractors had appeared.

However, if the animal were to compensate for the changes in sensory encoding by shifting its decision criterion, this could cancel the effects of sensory encoding on bias, making it seem as though there were no change in bias at all. Therefore, a lack of a change in bias does not guarantee a stable decision criterion. As we have shown, changes to sensory encoding that alter the target and distractor distributions in the same direction are common- 
place. For instance, the classic gain-change effects of both spatial and feature attention on neuronal activity should drive changes in both sensitivity and bias (Treue and Maunsell, 1996; Treue and Martínez-Trujillo, 1999). In contrast, changes to sensory encoding that proportionally change target and distractor distributions in opposite directions, such that the optimal criterion is stable, are less common.

We were initially surprised at the comparatively small effect of the optogenetic and stimulus manipulations on sensitivity compared with the large effects on bias. In particular, one might expect that changing stimulus contrast should affect sensitivity on the orientation discrimination task. However, similar effects have been observed with contrast manipulations in a go/no-go orientation discrimination task (Long et al., 2015), and we think that the observed effects can be largely explained by the computation that the mouse is using to perform the task. Our previous study suggests that the circuits downstream of $\mathrm{V} 1$ are monitoring the total firing rates of a population of target-responsive sensory neurons (Jin et al., 2019). When the firing rate of this population exceeds some threshold, a target is detected. The data presented here support this model: increases in contrast or ISI or optogenetic activation of $\mathrm{V} 1$ neurons increase $\mathrm{V} 1$ firing rates and so are mistaken for target orientations. Although the proposed decoder is suboptimal for discriminating orientations, we do not think our results are specific to this orientation discrimination task or limited to this particular decoder strategy. First, we also observe that decreases in firing rate in V1 increase bias in a contrast detection task in which a similar all positive sum decoder is feasible. Second, some optimal decoding strategies that compute the estimated orientation from the population activity, for instance through a likelihood function, are also sensitive to manipulations of excitability in sensory cortex because of changes in certainty, and thus may also affect measured bias (Stocker and Simoncelli, 2006).

We find that manipulating stimulus contrast and ISI induced relatively small effects on V1 activity, and therefore the predicted hit rate, compared with the effects observed behaviorally (Figs. $6 C$ vs $E, 7 C$ vs $E$ ). This discrepancy could be because of multiple factors. First, although we think we have identified the general computation used to perform this task, we cannot determine the precise weighting assigned to the neuronal population, and therefore the transformation between neuronal activity and behavior. Second, although V1 is required for this task, it is likely not the final visual processing stage. For instance, visual information in V1 is routed to higher visual cortical and areas (Wang and Burkhalter, 2007; Glickfeld et al., 2013; Glickfeld and Olsen, 2017); this process may transform representations to become more sensitive to contrast or ISI before feeding into the decoder. Indeed, our preliminary results suggest that some higher visual areas are also required for this task and they are more sensitive to adaptation than V1. Although the magnitude of the effects on firing rate and behavior differ, the fact that both measures change in the same direction across our manipulation conditions strongly supports our argument that changes in bias can be induced by changes in the sensory encoding.

Our data reveal that combining optogenetics and SDT to dissociate the sensory and cognitive contributions to perceptual decision-making in distinct brain circuits is not straightforward. This is because standard application of SDT conflates perceptual and response bias (Witt et al., 2015). Realizing this confound, some groups have designed tasks to support the dissociation of sensory and cognitive contributions through SDT analyses. One such approach is to take advantage of the temporal separability between these processes. For instance, studies normally use prestimulus cues to bias the behavioral choice, but by adding a poststimulus cue design one can better dissociate the effects of cue on sensory encoding and response bias (Bang and Rahnev, 2017). Other groups have taken advantage of clever stimulus design. For instance, using noisy stimulus sets to generate trial-by-trial variability enables experimenters to use regression-based approaches to measure stimulus sensitivity across conditions, and thereby dissociate perceptual and response bias (Wyart et al., 2012; Kloosterman et al., 2019). Together, these approaches can be combined with optogenetics to determine the extent to which brain areas and circuits contribute to the various stages of perceptual decision-making.

In conclusion, we have provided thorough experimental evidence that bias measured from SDT is not independent of sensory encoding, and in fact bias can be changed via directly manipulating sensory area V1. This introduces a conundrum that changes in bias can either result from sensory or cognitive mechanisms or both, which could be resolved via experimental design and new mathematical approaches. Our data are most consistent with a sensory role for $\mathrm{V} 1$, in which changes in sensory encoding are propagated to downstream decision-making areas and alter bias by shifting the optimal decision criterion relative to the actual decision criterion. Future work will be needed to determine whether V1 also contributes to cognitive aspects of the decisionmaking process.

\section{References}

Bang JW, Rahnev D (2017) Stimulus expectation alters decision criterion but not sensory signal in perceptual decision making. Sci Rep 7:17072.

Bashinski HS, Bacharach VR (1980) Enhancement of perceptual sensitivity as the result of selectively attending to spatial locations. Percept Psychophys 28:241-248.

Bennett C, Arroyo S, Hestrin S (2013) Subthreshold mechanisms underlying state-dependent modulation of visual responses. Neuron 80:350-357.

Carandini M, Churchland AK (2013) Probing perceptual decisions in rodents. Nat Neurosci 16:824-831.

Clifford CW, Webster MA, Stanley GB, Stocker AA, Kohn A, Sharpee TO, Schwartz O (2007) Visual adaptation: neural, psychological and computational aspects. Vision Res 47:3125-3131.

Crapse TB, Lau H, Basso MA (2018) A role for the superior colliculus in decision criteria. Neuron 97:181-194.e6.

de Gee JW, Colizoli O, Kloosterman NA, Knapen T, Nieuwenhuis S, Donner TH (2017) Dynamic modulation of decision biases by brainstem arousal systems. eLife 6:e23232.

Ditterich J, Mazurek ME, Shadlen MN (2003) Microstimulation of visual cortex affects the speed of perceptual decisions. Nat Neurosci 6:891-898.

Dragoi V, Sharma J, Sur M (2000) Adaptation-induced plasticity of orientation tuning in adult visual cortex. Neuron 28:287-298.

Fetsch CR, Odean NN, Jeurissen D, El-Shamayleh Y, Horwitz GD, Shadlen $\mathrm{MN}$, States U (2018) Focal optogenetic suppression in macaque area MT biases direction discrimination and decision confidence, but only transiently. eLife 7:e36523.

Glickfeld LL, Olsen SR (2017) Higher-order areas of the mouse visual cortex. Annu Rev Vis Sci 3:251-273.

Glickfeld LL, Andermann ML, Bonin V, Reid RC (2013) Cortico-cortical projections in mouse visual cortex are functionally target specific. Nat Neurosci 16:219-226.

Gold JI, Shadlen MN (2007) The neural basis of decision making. Annu Rev Neurosci 30:535-574

Green DM, Swets J (1966) Signal detection theory and psychophysics. New York: Wiley.

Grier JB (1971) Nonparametric indexes for sensitivity and bias: computing formulas. Psychol Bull 75:424-429.

Grove PM, Ashton J, Kawachi Y, Sakurai K (2012) Auditory transients do not affect visual sensitivity in discriminating between objective streaming and bouncing events. J Vis 12:5 1-11.

Hanks TD, Summerfield C (2017) Perceptual decision making in rodents, monkeys, and humans. Neuron 93:15-31. 
Herman JP, Katz LN, Krauzlis RJ (2018) Midbrain activity can explain perceptual decisions during an attention task. Nat Neurosci 21:1651-1655.

Hodos W (1970) Nonparametric index of response bias for use in detection and recognition experiments. Psychol Bull 74:351-354.

Iemi L, Chaumon M, Crouzet SM, Busch NA (2017) Spontaneous neural oscillations bias perception by modulating baseline excitability. J Neurosci 37:807-819.

Jin M, Beck JM, Glickfeld LL (2019) Neuronal adaptation reveals a suboptimal decoding of orientation tuned populations in the mouse visual cortex mouse visual cortex. J Neurosci. Advance online publication. Retrieved March 4, 2019. doi:10.1523/JNEUROSCI.3172-18.2019.

Jones PR, Moore DR, Shub DE, Amitay S (2015) The role of response bias in perceptual learning. J Exp Psychol Learn Mem Cogn 41:1456-1470.

Jurjut O, Georgieva P, Busse L, Katzner S (2017) Learning enhances sensory processing in mouse V1 before improving behavior. J Neurosci 37: $6460-6474$.

Kang I, Maunsell JH (2012) Potential confounds in estimating trial-to-trial correlations between neuronal response and behavior using choice probabilities. J Neurophysiol 108:3403-3415.

Kloosterman NA, de Gee JW, Werkle-Bergner M, Lindenberger U, Garrett DD, Fahrenfort JJ (2019) Humans strategically shift decision bias by flexibly adjusting sensory evidence accumulation. eLife 8:e37321.

Kok P, Brouwer GJ, van Gerven MA, de Lange FP (2013) Prior expectations bias sensory representations in visual cortex. J Neurosci 33:16275-16284.

Long M, Jiang W, Liu D, Yao H (2015) Contrast-dependent orientation discrimination in the mouse. Sci Rep 5:15830.

Luo TZ, Maunsell JH (2015) Neuronal modulations in visual cortex are associated with only one of multiple components of attention. Neuron 86:1182-1188.

Luo TZ, Maunsell JHR (2018) Attentional changes in either criterion or sensitivity are associated with robust modulations in lateral prefrontal cortex. Neuron 97:1382-1393.e7.

Macmillan NA, Kaplan HL (1985) Detection theory analysis of group data: estimating sensitivity from average hit and false-alarm rates. Psychol Bull 98:185-199.

McDonald JJ, Teder-Sälejärvi WA, Hillyard SA (2000) Involuntary orienting to sound improves visual perception. Nature 407:906-908.

Müller JR, Metha AB, Krauskopf J, Lennie P (1999) Rapid adaptation in visual cortex to the structure of images. Science 285:1405-1408.

Ni AM, Ruff DA, Alberts JJ, Symmonds J, Cohen MR (2018) Learning and attention reveal a general relationship between neuronal variability and perception. Science 359:463-465.

Nienborg H, Hasenstaub A, Nauhaus I, Taniguchi H, Huang ZJ, Callaway EM
(2013) Contrast dependence and differential contributions from somatostatin- and parvalbumin-expressing neurons to spatial integration in mouse V1. J Neurosci 33:11145-11154.

Ohzawa I, Sclar G, Freeman RD (1982) Contrast gain control in the cat viusal cortex. Nature 298:266-268.

Pinto L, Goard MJ, Estandian D, Xu M, Kwan AC, Lee SH, Harrison TC, Feng G, Dan Y (2013) Fast modulation of visual perception by basal forebrain cholinergic neurons. Nat Neurosci 16:1857-1863.

Pollack I, Norman DA (1964) A non-parametric analysis of recognition experiments. Psychon Sci 1:125-126.

Romo R, de Lafuente V (2013) Conversion of sensory signals into perceptual decisions. Prog Neurobiol 103:41-75.

Salzman CD, Britten KH, Newsome WT (1990) Cortical microstimulation influences perceptual judgements of motion direction. Nature 346:174177.

Sengpiel F, Sen A, Blakemore C (1997) Characteristics of surround inhibition in cat area 17. Exp Brain Res 116:216-228.

Stanislaw H, Todorov N (1999) Calculation of signal detection theory measures. Behav Res Methods Instrum Comput 31:137-149.

Stocker AA, Simoncelli EP (2006) Noise characteristics and prior expectations in human visual speed perception. Nat Neurosci 9:578-585.

Treue S, Martínez-Trujillo JC (1999) Feature-based attention influences motion processing gain in macaque visual cortex. Nature 399:575-579.

Treue S, Maunsell JH (1996) Attentional modulation of visual motion processing inn cortical areas MT and MST. Nature 382:539-541.

van Vugt B, Dagnino B, Vartak D, Safaai H, Panzeri S, Dehaene S, Roelfsema PR (2018) The threshold for conscious report: signal loss and response bias in visual and frontal cortex. Science 360:537-542.

Vintch B, Gardner JL (2014) Cortical correlates of human motion perception biases. J Neurosci 34:2592-2604.

Wang L, Rangarajan KV, Gerfen CR, Krauzlis RJ (2018) Activation of striatal neurons causes a perceptual decision bias during visual change detection in mice. Neuron 97:1369-1381.e5.

Wang Q, Burkhalter A (2007) Area map of mouse visual cortex. J Comp Neurol 502:339-357.

White CN, Mumford JA, Poldrack RA (2012) Perceptual criteria in the human brain. J Neurosci 32:16716-16724.

Witt JK, Taylor JE, Sugovic M, Wixted JT (2015) Signal detection measures cannot distinguish perceptual biases from response biases. Perception 44:289-300.

Wyart V, Nobre AC, Summerfield C (2012) Dissociable prior influences of signal probability and relevance on visual contrast sensitivity. Proc Natl Acad Sci U S A 109:3593-3598. 